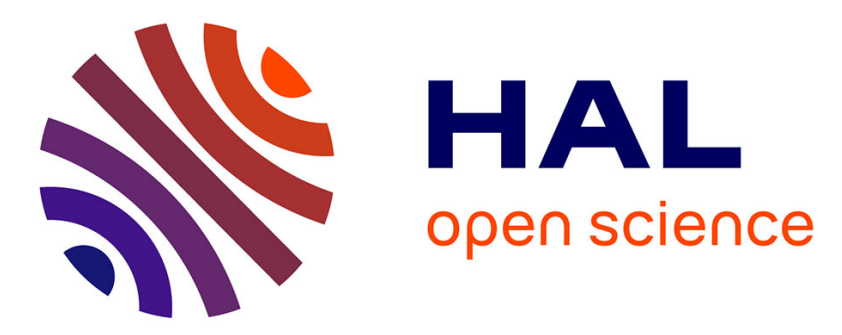

\title{
Explicit dynamic analysis of sheet metal forming processes using linear prismatic and hexahedral solid-shell elements
}

Peng Wang, Hocine Chalal, Farid Abed-Meraim

\section{- To cite this version:}

Peng Wang, Hocine Chalal, Farid Abed-Meraim. Explicit dynamic analysis of sheet metal forming processes using linear prismatic and hexahedral solid-shell elements. Engineering Computations, 2017, 34 (5), pp.1413-1445. 10.1108/EC-04-2016-0150 . hal-02386217

\section{HAL Id: hal-02386217 https://hal.science/hal-02386217}

Submitted on 29 Nov 2019

HAL is a multi-disciplinary open access archive for the deposit and dissemination of scientific research documents, whether they are published or not. The documents may come from teaching and research institutions in France or abroad, or from public or private research centers.
L'archive ouverte pluridisciplinaire HAL, est destinée au dépôt et à la diffusion de documents scientifiques de niveau recherche, publiés ou non, émanant des établissements d'enseignement et de recherche français ou étrangers, des laboratoires publics ou privés. 


\title{
Explicit dynamic analysis of sheet metal forming processes using linear prismatic and hexahedral solid-shell elements
}

\begin{abstract}
Propose - The purpose of this paper is to propose two linear solid-shell finite elements, a sixnode prismatic element denoted SHB6-EXP and an eight-node hexahedral element denoted SHB8PS-EXP, for the three-dimensional modeling of thin structures in the context of explicit dynamic analysis.

Design/methodology/approach - These two linear solid-shell elements are formulated based on a purely three-dimensional approach, with displacements as the only degrees of freedom. To prevent various locking phenomena, a reduced-integration scheme is used along with the assumedstrain method. The resulting formulations are computationally efficient, since only a single layer of elements with an arbitrary number of through-thickness integration points is required to model 3D thin structures.
\end{abstract}

Findings - Via the VUEL user-element subroutines, the performance of these elements is assessed through a set of selective and representative dynamic elasto-plastic benchmark tests, impact-type problems and deep drawing processes involving complex non-linear loading paths, anisotropic plasticity and double-sided contact. The obtained numerical results demonstrate the good performance of the SHB-EXP elements in the modeling of 3D thin structures, with only a single element layer and few integration points in the thickness direction.

Originality/value - The extension of the SHB-EXP solid-shell formulations to large-strain anisotropic plasticity enlarges their application range to a wide variety of dynamic elasto-plastic problems and sheet metal forming simulations. All simulation results reveal that the numerical strategy adopted in this paper can efficiently prevent the various locking phenomena that commonly occur in the 3D modeling of thin structural problems.

Keywords: Finite element, Solid-shell concept, Explicit dynamic analysis, Anisotropic elastoplasticity, Impact problems, Sheet metal forming 


\section{Introduction}

The finite element (FE) simulation of sheet metal forming processes has become an indispensable tool in the design and manufacturing of modern products in many industries. Such a simulation tool greatly assists designers and engineers in predicting the final shape of products and optimizing forming setups, by replacing a number of expensive and time-consuming experimental tests. However, the accuracy and efficiency of the FE simulation should be guaranteed in order to obtain reliable predictions. Traditionally, for the simulation of thin structures, conventional shell elements are used or alternatively low-order solid elements, when three-dimensional effects need to be accounted for. However, conventional shell elements show limitations in the simulation of a number of sheet metal forming processes, while low-order solid elements suffer from various locking phenomena. In this regard, much effort has been devoted in the literature to establish accurate and efficient finite element formulations.

The membrane finite element (Sukhomlinov et al., 1992; Huh et al., 1994; Huh and Choi, 1999) has been widely used, due to its computational efficiency in the simulation of bending as well as stretching-dominated sheet metal forming problems. In order to obtain more accurate results, particular attention has been paid in the literature to the development of shell elements for the modeling of thin structures. For instance, Guo et al. (2002) proposed an efficient shell element based on the discrete Kirchhoff assumption for the simulation of springback in sheet metal forming processes, while Lu et al. (2006) developed their own degenerated shell element based on Mindlin-Reissner's theory. Compared to membrane elements, shell elements offer better accuracy for modeling bending effects in thin structures. However, the formulation of classical shell elements is typically based on the assumption of plane-stress conditions, which limits their application in sheet metal forming simulation. Further, they cannot account for thickness variations, since only the mid-plane of the sheet is modeled, which makes the double-sided contact difficult to handle.

Concurrently, continuum solid elements allow more realistic modeling for a number of structural problems thanks to their three-dimensional formulation, thus avoiding geometric (midplane) or kinematics assumptions as well as constitutive (plane-stress) restrictions. However, in the simulation of thin structures, the use of solid elements involves meshes with too many elements, which is partly attributable to element aspect ratio limitations as well as locking effects 
in low-order formulations. In addition, several layers of solid elements are required in the thickness direction in order to accurately describe the various non-linear phenomena, which considerably increases the computational cost of the simulations.

More recently, the concept of solid--shell elements has emerged, which represents nowadays an interesting alternative to conventional solid and shell elements, in particular for the simulation of sheet metal forming processes. In fact, solid--shell elements combine the advantages of both solid and shell formulations. Their main key features, which make them very attractive, may be summarized as follows: the use of fully three-dimensional constitutive laws, without plane-stress restrictions; easy connection with conventional solid elements, since displacements are the only degrees of freedom; direct calculation of thickness variations, as this is based on physical nodes; automatic consideration of double-sided contact; ability to accurately model thin structures with only a single element layer and few integration points in the thickness direction.

In the past few decades, various solid-shell elements have been developed in the literature, on the basis of different approaches (Cho et al., 1998; Hauptmann and Schweizerhof, 1998; Puso, 2000; Sze and Yao, 2000; Abed-Meraim and Combescure, 2002; Parente et al., 2006; Reese, 2007; Cardoso et al., 2008; Abed-Meraim and Combescure, 2009; Schwarze and Reese, 2009; Li et al., 2011; Trinh et al., 2011; Edem and Gosling, 2013; Flores, 2013; Pagani et al., 2014). Most of these formulations have been established based on the assumed strain method (ASM), the enhanced assumed strain (EAS) approach, or the assumed natural strain (ANS) concept. Recent formulations also combine some of these techniques in order to further eliminate all kinds of spurious mechanisms (e.g., rank deficiencies) and locking phenomena. The key idea on which these approaches are based is to enrich the kinematics (through additional enhanced/assumed strain fields), in the aim of eliminating various locking effects (membrane, shear, volumetric, thickness ...). The reduced-integration rule is sometimes additionally used to alleviate some locking effects, but this may lead to rank deficiency, which requires the element formulation to be stabilized against hourglass (zero-energy) modes.

In this paper, two linear solid-shell element formulations are proposed for the explicit dynamic analysis of structural problems and sheet metal forming processes. These explicit dynamic solid-shell versions are derived by extending their quasi-static counterparts, and consist of a sixnode prismatic element, denoted SHB6-EXP, and an eight-node hexahedral element, denoted 
SHB8PS-EXP. Their formulation is based on a fully three-dimensional approach, in which the nodal displacements are the only degrees of freedom, in conjunction with the reduced-integration technique and the assumed-strain method to alleviate the locking problems. The SHB8PS-EXP element is an extension, to the explicit dynamic framework, of the previously developed quasistatic version (Abed-Meraim and Combescure, 2009). Note that an explicit dynamic version of this element has been formulated earlier to deal with impact problems (Abed-Meraim and Combescure, 2002); however, it was restricted to isotropic elasto-plastic constitutive models. In addition, selective benchmark tests revealed that it suffers from several locking phenomena. The motivation of the improved subsequent formulation (Abed-Meraim and Combescure, 2009), although within a quasi-static framework, was to eliminate the above-mentioned locking problems through enhanced assumed-strain fields and a new stabilization scheme for the control of hourglass modes. It is this latter version that is extended in the current work to the explicit dynamic analysis of thin structural problems, through its coupling with advanced anisotropic behavior models. As to the SHB6-EXP element, its dynamic explicit formulation is obtained here, for the first time, by extending the quasi-static version developed in Trinh et al. (2011). In the latter contribution, various popular benchmark tests have been used to assess the performance of this solid-shell element in the framework of quasi-static small-strain analysis and linear elastic problems. In the current work, the explicit dynamic extensions SHB8PS-EXP and SHB6-EXP, which are provided with a simple lumped mass matrix, are formulated within the framework of large-strain anisotropic plasticity and implemented into the explicit dynamic code ABAQUS for the simulation of impact problems and complex and challenging sheet metal forming processes that are difficult to perform using quasi-static solvers.

The remainder of the paper is organized as follows. The explicit dynamic formulation of the SHB-EXP solid-shell elements is first presented in Section 2. Then, the performance of the SHBEXP elements is evaluated in Section 3 through various benchmark tests, including selective and representative dynamic/impact problems. To assess the performance of the SHB-EXP elements in complex highly non-linear test problems, the proposed solid-shell elements are applied in Section 4 to the simulation of three deep drawing processes, involving geometric non-linearities, anisotropic elasto-plastic behavior, and double-sided contact. All of the numerical results obtained with the SHB-EXP elements are compared, on the one hand, to those provided by state-of-the-art 
elements available in ABAQUS, using equivalent mesh refinement, and, on the other hand, to reference solutions and experimental results taken from the literature. Finally, the main discussions and concluding remarks are summarized in Section 5.

\section{Formulation of linear solid-shell elements}

This section provides the formulation of the SHB8PS-EXP and SHB6-EXP solid-shell elements and the associated new enhancements in the context of explicit dynamic analysis. The interested reader may refer to Abed-Meraim and Combescure (2009) and Trinh et al. (2011), respectively, for their detailed implicit quasi-static formulations.

\subsection{General formulation of the SHB6-EXP and SHB8PS-EXP elements}

The reference geometry for the six-node prismatic solid-shell element SHB6-EXP and for the eight-node hexahedral solid-shell element SHB8PS-EXP is shown in Figures 1(a) and (b), respectively, where a special direction $\zeta$ is chosen to represent the thickness direction. Along this direction, a user-defined arbitrary number of integration points are arranged. This choice aims at providing the proposed elements, although geometrically three-dimensional, with some shell features. It also allows alleviating some locking phenomena and improving the computational efficiency of the elements, by using only a single element layer in the simulation of thin structures.

(a)

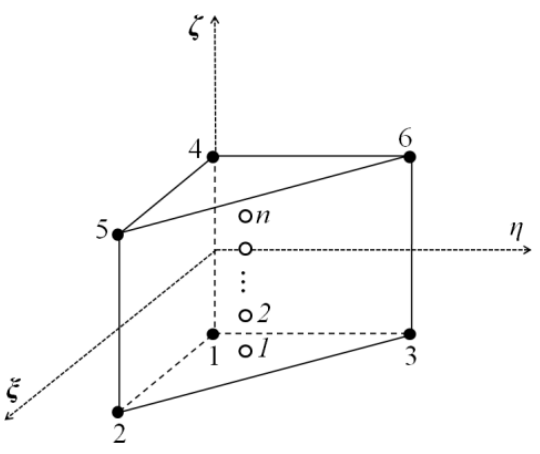

(b)

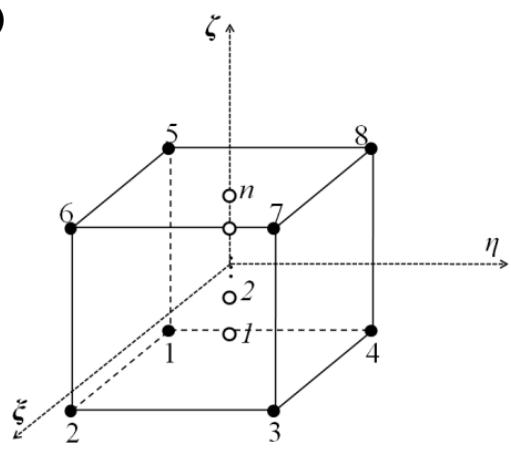

Figure 1. Geometry of the SHB-EXP elements and location of their integration points in the reference coordinate frame: (a) SHB6-EXP element and (b) SHB8PS-EXP element

2.1.1 Linear interpolation of the elements. The current SHB-EXP solid-shell elements are isoparametric elements, and use the classical linear shape functions for continuum standard hexahedral and prismatic elements. Based on this three-dimensional formulation, the coordinates 
$x_{i}$ and velocities $v_{i}$ inside an element are interpolated using the linear shape functions $N_{I}$, the nodal coordinates $x_{i I}$ and the nodal velocities $v_{i I}$ as

$$
\begin{gathered}
x_{i}=x_{i I} N_{I}(\xi, \eta, \zeta)=\sum_{I=1}^{n} N_{I}(\xi, \eta, \zeta) x_{i I}, \\
v_{i}=v_{i I} N_{I}(\xi, \eta, \zeta)
\end{gathered}
$$

where the lowercase subscript $i$ varies from 1 to 3 and represents the spatial coordinate directions, while the uppercase subscript $I$ varies from 1 to $n$, where $n$ denotes the number of element nodes (i.e., $n=6$ for the SHB6-EXP element, and $n=8$ for the SHB8PS-EXP element). In what follows, the convention of implied summation on repeated indices will be adopted, as in Eq. (2) above.

2.1.2 Derivation of discrete gradient operator. Combining Eqs. (1) and (2) allows us to derive the complete expression of the velocity field $v_{i}$ as follows:

$$
v_{i}=a_{0 i}+a_{1 i} x_{1}+a_{2 i} x_{2}+a_{3 i} x_{3}+c_{1 i} h_{1}+\cdots+c_{\alpha i} h_{\alpha} \text { with } i=1,2,3,
$$

where the functions $h_{\alpha}$ are specific to each of the SHB-EXP elements. For instance, subscript $\alpha$ varies from 1 to 4 for the SHB8PS-EXP element, while it varies from 1 to 2 for the SHB6-EXP element. Functions $h_{\alpha}$ have the following expressions:

$$
h_{1}=\eta \zeta, \quad h_{2}=\zeta \xi, \quad h_{3}=\xi \eta, \quad h_{4}=\xi \eta \zeta .
$$

Applying, in particular, the velocity field expansion (3) to the element nodes, the nodal velocity vectors $\dot{\mathbf{d}}_{i}$, associated with the hexahedral and prismatic SHB-EXP elements, can be expressed in the following compact form:

$$
\dot{\mathbf{d}}_{i}=a_{0 i} \mathbf{s}+a_{1 i} \mathbf{x}_{1}+a_{2 i} \mathbf{x}_{2}+a_{3 i} \mathbf{x}_{3}+c_{1 i} \mathbf{h}_{1}+\cdots+c_{\alpha i} \mathbf{h}_{\alpha} \quad \text { with } i=1,2,3,
$$

where $\mathbf{x}_{i}, i=1,2,3$, represent the nodal coordinate vectors, while the detailed expressions of vectors $\mathbf{s}$ and $\mathbf{h}_{\alpha}$ can be found in Trinh et al. (2011) for the six-node prismatic element, and in Abed-Meraim and Combescure (2009) for the eight-node hexahedral element.

By introducing the derivatives of the shape functions $\mathbf{b}_{i}=\mathbf{N}_{, i}(0,0,0), i=1,2,3$, also known as Hallquist's vectors (Hallquist, 1983), and using some orthogonality relations that are easy to demonstrate, the expression of the unknown constants in Eqs. (3) and (5) can be obtained (see, e.g., Abed-Meraim and Combescure, 2009; Trinh et al., 2011) 


$$
\left\{\begin{array}{l}
a_{j i}=\mathbf{b}_{j}^{T} \cdot \dot{\mathbf{d}}_{i}, \quad c_{\alpha i}=\boldsymbol{\gamma}_{\alpha}^{T} \cdot \dot{\mathbf{d}}_{i} \\
\text { where: } \boldsymbol{\gamma}_{\alpha}=\frac{1}{k}\left[\mathbf{h}_{\alpha}-\sum_{j=1}^{3}\left(\mathbf{h}_{\alpha}^{T} \cdot \mathbf{x}_{j}\right) \mathbf{b}_{j}\right]
\end{array}\right.
$$

with $k=2$ for the SHB6-EXP element, and $k=8$ for the SHB8PS-EXP element. Substituting the expressions of these constants into Eq. (3), the velocity field can be rewritten in the following more convenient form:

$$
v_{i}=a_{0 i}+\left(x_{1} \mathbf{b}_{1}^{T}+x_{2} \mathbf{b}_{2}^{T}+x_{3} \mathbf{b}_{3}^{T}+h_{1} \boldsymbol{\gamma}_{1}^{T}+\cdots+h_{\alpha} \boldsymbol{\gamma}_{\alpha}^{T}\right) \cdot \dot{\mathbf{d}}_{i} .
$$

The velocity gradient is then obtained by differentiating the above expression with respect to $x_{j}$ as follows:

$$
v_{i, j}=\left(\mathbf{b}_{j}^{T}+\sum_{\alpha} h_{\alpha, j} \gamma_{\alpha}^{T}\right) \cdot \dot{\mathbf{d}}_{i}=\left(\mathbf{b}_{j}^{T}+h_{\alpha, j} \boldsymbol{\gamma}_{\alpha}^{T}\right) \cdot \dot{\mathbf{d}}_{i} .
$$

Finally, the vector form of the velocity gradient operator can be expressed as follows:

$$
\nabla_{s}(\mathbf{v})=\left[\begin{array}{c}
v_{x, x} \\
v_{y, y} \\
v_{z, z} \\
v_{x, y}+v_{y, x} \\
v_{y, z}+v_{z, y} \\
v_{x, z}+v_{z, x}
\end{array}\right]=\mathbf{B} \cdot \dot{\mathbf{d}}=\mathbf{B} \cdot\left[\begin{array}{c}
\dot{\mathbf{d}}_{x} \\
\dot{\mathbf{d}}_{y} \\
\dot{\mathbf{d}}_{z}
\end{array}\right],
$$

where the discrete gradient operator $\mathbf{B}$ takes the following matrix form:

$$
\mathbf{B}=\left[\begin{array}{ccc}
\mathbf{b}_{x}^{T}+h_{\alpha, x} \boldsymbol{\gamma}_{\alpha}^{T} & 0 & 0 \\
0 & \mathbf{b}_{y}^{T}+h_{\alpha, y} \boldsymbol{\gamma}_{\alpha}^{T} & 0 \\
0 & 0 & \mathbf{b}_{z}^{T}+h_{\alpha, z} \boldsymbol{\gamma}_{\alpha}^{T} \\
\mathbf{b}_{y}^{T}+h_{\alpha, y} \boldsymbol{\gamma}_{\alpha}^{T} & \mathbf{b}_{x}^{T}+h_{\alpha, x} \boldsymbol{\gamma}_{\alpha}^{T} & 0 \\
0 & \mathbf{b}_{z}^{T}+h_{\alpha, z} \boldsymbol{\gamma}_{\alpha}^{T} & \mathbf{b}_{y}^{T}+h_{\alpha, y} \boldsymbol{\gamma}_{\alpha}^{T} \\
\mathbf{b}_{z}^{T}+h_{\alpha, z} \boldsymbol{\gamma}_{\alpha}^{T} & 0 & \mathbf{b}_{x}^{T}+h_{\alpha, x} \boldsymbol{\gamma}_{\alpha}^{T}
\end{array}\right] .
$$

2.1.3 Hu-Washizu principle and internal forces. The assumed-strain method used in the formulation of the SHB-EXP solid-shell elements is based on the simplified form of the $\mathrm{Hu}-$ Washizu mixed variational principle, as proposed by Simo and Hughes (1986)

$$
\delta \pi(\dot{\overline{\boldsymbol{\varepsilon}}})=\int_{\Omega_{e}} \delta \dot{\overline{\boldsymbol{\varepsilon}}}^{T} \cdot \boldsymbol{\sigma} d \Omega-\delta \dot{\mathbf{d}}^{T} \cdot \mathbf{f}^{\text {ext }}=0
$$


where $\delta$ represents a variation, $\dot{\bar{\varepsilon}}$ the assumed-strain rate, $\boldsymbol{\sigma}$ the stress state obtained by the constitutive law, $\dot{\mathbf{d}}$ the nodal velocities, and $\mathbf{f}^{\text {ext }}$ the external nodal forces. Such a simplified principle is very convenient, because it only involves the interpolation of the velocity and of the assumed-strain field. The latter may be expressed over the element using an appropriate projection, which will be denoted $\overline{\mathbf{B}}$, of the original discrete gradient operator $\mathbf{B}$

$$
\dot{\overline{\boldsymbol{\varepsilon}}}(x, t)=\overline{\mathbf{B}}(x) \cdot \dot{\mathbf{d}}(t) .
$$

By substituting Eq. (12) into the simplified expression (11) of the $\mathrm{Hu}$-Washizu mixed variational principle, the following equation is obtained:

$$
\delta \dot{\mathbf{d}}^{T} \cdot\left(\int_{\Omega_{e}} \overline{\mathbf{B}}^{T} \cdot \boldsymbol{\sigma} d \Omega-\mathbf{f}^{e x t}\right)=0 .
$$

Because $\delta \dot{\mathbf{d}}$ can be chosen arbitrarily, the above equation allows the expression of the internal force vector to be derived as follows:

$$
\mathbf{f}^{\mathrm{int}}=\int_{\Omega_{e}} \overline{\mathbf{B}}^{T} \cdot \boldsymbol{\sigma}(\dot{\overline{\boldsymbol{\varepsilon}}}) d \Omega .
$$

In addition, due to the particular location along the same line of the integration points, it has been shown in Abed-Meraim and Combescure (2009) that the above formulation of the eight-node hexahedral solid-shell element generates six hourglass modes. These spurious zero-energy modes are controlled by applying an efficient stabilization technique, following the assumed-strain approach proposed in Belytschko and Bindeman (1993). The resulting internal force vector is obtained by adding to the usual internal forces (see Eq. (14)) a stabilization term, as follows:

$$
\mathbf{f}^{\text {int }}=\int_{\Omega_{e}} \overline{\mathbf{B}}^{T} \cdot \boldsymbol{\sigma}(\dot{\bar{\varepsilon}}) d \Omega+\mathbf{f}^{\mathrm{STAB}} .
$$

More details on the derivation of the expression of the stabilization forces $\mathbf{f}^{\mathrm{STAB}}$ in the case of the eight-node hexahedral solid-shell element can be found in Abed-Meraim and Combescure (2002). It is worth noting that the formulation of the six-node prismatic solid-shell element does not induce hourglass modes and, therefore, no additional stabilization terms are needed for the calculation of the associated internal forces. However, an appropriate projection of the strains for the SHB6-EXP element is required to eliminate some locking phenomena (Trinh et al., 2011).

2.1.4 Constitutive equations. The formulation of the SHB-EXP solid-shell elements requires resorting to several local frames in order to perform the entire calculations (see Figure 2 for illustration). These local physical or material coordinate systems are motivated by the computation 
of the elasticity law, the material plastic anisotropy, or the stabilization terms. For instance, the socalled "element frame" is introduced for the definition of the material elastic properties. Accordingly, the elasticity law is specified in such a local physical coordinate system, which corresponds to the element mid-plane associated with the $\zeta$-coordinate of each integration point. In these local coordinate systems, which are attached to the integration points of the element, the classical three-dimensional elasticity matrix is modified so that plane-stress conditions are approached. This modification in the elasticity matrix, which has been validated in the earlier quasi-static formulations of the SHB elements (see, e.g., Abed-Meraim and Combescure, 2009; Trinh et al., 2011), allows enhancing the performance of the proposed elements with regard to thickness locking. Such a specific improved plane-stress type elasticity matrix $\mathbf{C}^{\text {ele }}$ is defined as follows:

$$
\mathbf{C}^{\mathrm{ele}}=\left[\begin{array}{cccccc}
\bar{\lambda}+2 \mu & \bar{\lambda} & 0 & 0 & 0 & 0 \\
\bar{\lambda} & \bar{\lambda}+2 \mu & 0 & 0 & 0 & 0 \\
0 & 0 & E & 0 & 0 & 0 \\
0 & 0 & 0 & \mu & 0 & 0 \\
0 & 0 & 0 & 0 & \mu & 0 \\
0 & 0 & 0 & 0 & 0 & \mu
\end{array}\right] \text { with } \mu=\frac{E}{2(1+v)} \text { and } \bar{\lambda}=\frac{E v}{1-v^{2}}
$$

where $E$ and $v$ are Young's modulus and Poisson's ratio, respectively.

The so-called "material frame" is another local physical coordinate system, which is introduced to account for the initial plastic anisotropy of the material and its evolution in the course of deformation. The time integration of the large-strain anisotropic elasto-plastic constitutive equations, which is achieved at each integration point, also uses this local material frame in order to satisfy the objectivity (material invariance) requirements.

Finally, a third local coordinate system, designated as "co-rotational frame", is defined at the element level in order to simplify the calculation of the stabilization terms involved in the expression of the internal forces, in the particular case of SHB8PS-EXP element.

For a given rotation matrix $\mathbf{R}$, corresponding to one of the three local coordinate systems described above, the tensor variables can be transformed from the global coordinate system into the local coordinate frame by using the following classical formulas:

$$
\begin{gathered}
\mathbf{a}^{\text {loc }}=\mathbf{R}^{\mathrm{T}} \cdot \mathbf{a}^{\text {glo }} \cdot \mathbf{R} \\
\mathbf{A}^{\text {loc }}=\mathbf{R}^{\mathrm{T}} \cdot \mathbf{R}^{\mathrm{T}} \cdot \mathbf{A}^{\text {glo }} \cdot \mathbf{R} \cdot \mathbf{R} \\
9
\end{gathered}
$$


where $\mathbf{a}^{\text {loc }}$ and $\mathbf{A}^{\text {loc }}$ represent second-order, respectively, fourth-order tensors expressed in the local coordinate system, while $\mathbf{a}^{\text {glo }}$ and $\mathbf{A}^{\text {glo }}$ are their expressions in the global coordinate frame. Using the indicial notation, the above equation can be rewritten in the following equivalent form:

$$
\begin{aligned}
& a_{i j}^{\mathrm{loc}}=R_{p i} R_{q j} a_{p q}^{\mathrm{glo}} \\
& A_{i j k l}^{\mathrm{loc}}=R_{p i} R_{q j} R_{r k} R_{s l} A_{p q r s}^{\mathrm{glo}},
\end{aligned}
$$

where the lowercase subscripts $i, j, k, l, p, q, r$ and $s$ vary from 1 to 3 .

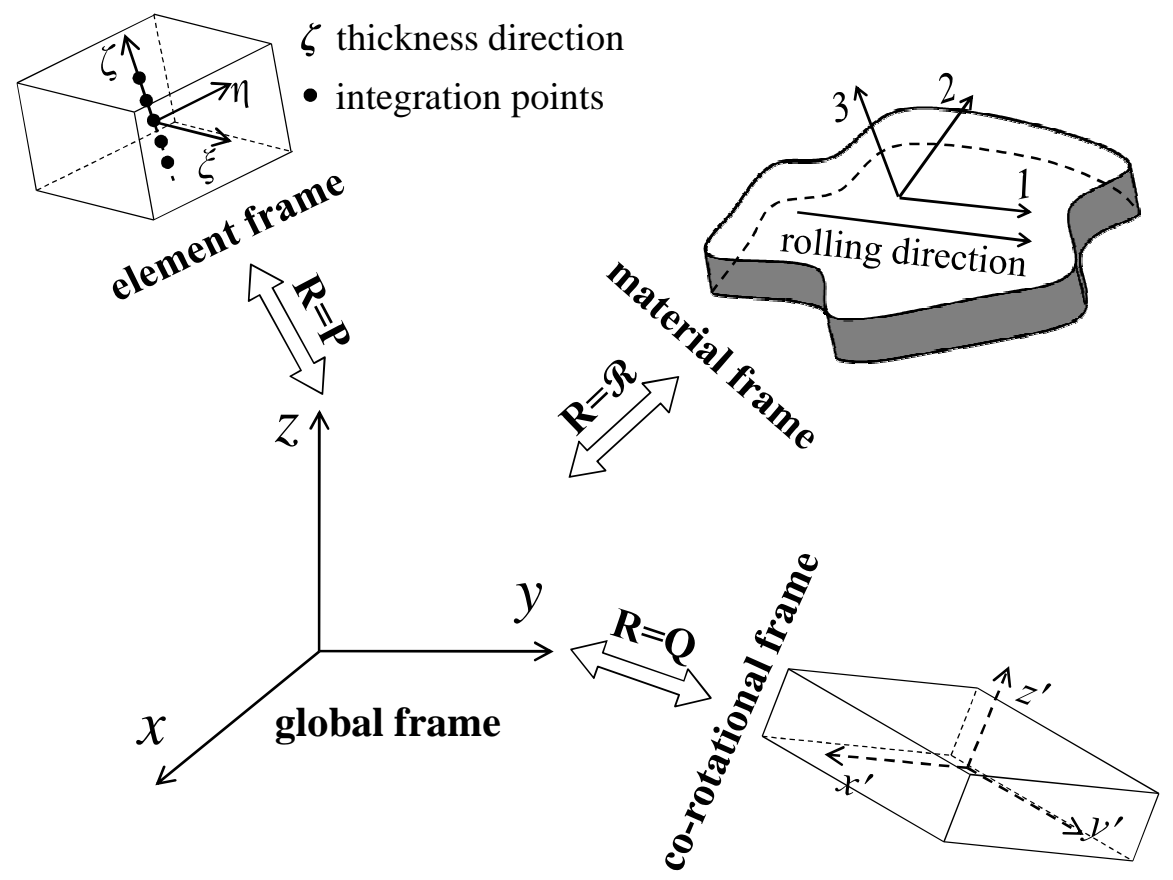

Figure 2. Illustration of the local coordinate systems, and the associated rotation matrices, used in the formulation of SHB-EXP solid-shell elements

As stated before, in the formulation of the SHB-EXP solid-shell elements, the rate constitutive equations are integrated in the local material frame in order to ensure material objectivity within the large-strain framework. All constitutive equations are implemented into the finite element software package ABAQUS/Explicit, within the framework of large plastic deformations, using an independent VUMAT-like user-material subroutine. The latter is called by the user-element subroutines associated with the SHB-EXP elements to update the stress state and other internal variables, which allows easy and modular coupling with any new constitutive model, independently of the element formulation. In what follows, the constitutive equations are 
presented in the local material frame described above. In this material frame, the rate form of the Cauchy stress-strain relationship is expressed using the hypoelastic law defined by

$$
\dot{\boldsymbol{\sigma}}=\mathbf{C}:\left(\mathbf{D}-\mathbf{D}^{p}\right)
$$

where $\mathbf{C}$ is the fourth-order elasticity tensor, which is obtained by rotating the elasticity tensor $\mathbf{C}^{\text {ele }}$ (see Eq. (16)) from the element frame to the material frame. The strain rate tensor $\mathbf{D}$ is additively decomposed into an elastic part $\mathbf{D}^{e}$ and a plastic part $\mathbf{D}^{p}$. The latter is defined by an associative plastic flow rule

$$
\mathbf{D}^{p}=\dot{\lambda} \frac{\partial f}{\partial \boldsymbol{\sigma}}=\dot{\lambda} \mathbf{V}
$$

where $f$ represents the plastic yield surface, and $\mathbf{V}$ is the flow direction normal to the yield surface. The plastic multiplier $\dot{\lambda}$ in Eq. (20) is determined by the consistency condition.

In this work, the anisotropic plastic behavior of the material is taken into account by considering Hill's quadratic anisotropic yield criterion (Hill, 1948). The corresponding plastic yield function $f$ is written in the following form:

$$
f=\bar{\sigma}_{e q}-Y \leq 0,
$$

where $\bar{\sigma}_{e q}=\sqrt{\left(\boldsymbol{\sigma}^{\prime}-\boldsymbol{\alpha}\right): \mathbf{H}:\left(\boldsymbol{\sigma}^{\prime}-\boldsymbol{\alpha}\right)}$ is the equivalent stress, and $\boldsymbol{\sigma}^{\prime}$ is the deviatoric part of the Cauchy stress tensor. The fourth-order tensor $\mathbf{H}$ contains the six anisotropy coefficients of Hill' 48 quadratic yield criterion. The isotropic hardening of the material is described by the scalar variable $Y$, which characterizes the size of the yield surface, while kinematic hardening is represented by the back-stress tensor $\boldsymbol{\alpha}$.

For the above-described constitutive equations, it can be shown that the set of internal variables is governed by a generic differential equation of the form

$$
\dot{\mathbf{x}}=\mathbf{U}_{\mathbf{x}}(\mathbf{x}),
$$

where vector $\mathbf{x}$ contains all variables of the model that need to be updated. The function $\mathbf{U}_{\mathbf{x}}(\mathbf{x})$ depends on the specific equations that govern the evolution of internal variables, which can be either scalar or tensorial according to the type of variables. This general form (22) allows encompassing various hardening descriptions as well as more advanced yield surface models. For instance, the $\mathrm{U}_{Y}$ functions corresponding to the evolution of isotropic hardening, for the three 
different hardening models considered in this work, are summarized in Table 1 . In this table, $\sigma_{0}$ and $\bar{\varepsilon}$ are the initial yield stress and equivalent plastic strain, respectively, while $K, \varepsilon_{0}$, and $N$ represent hardening parameters.

Table 1. Definition of the isotropic hardening models considered and their evolution laws.

Using the above constitutive equations and the consistency condition $\dot{f}=0$, the expression of the plastic multiplier $\dot{\lambda}$ is derived as follows:

$$
\dot{\lambda}=\frac{\mathbf{V}: \mathbf{C}: \mathbf{D}}{\mathbf{V}: \mathbf{C}: \mathbf{V}+\mathbf{V}: \mathbf{U}_{\alpha}+\mathbf{U}_{Y}}
$$

2.1.5 The basic expression of the mass matrix. In this work, the SHB-EXP elements are implemented into the finite element software package ABAQUS/Explicit, within the framework of explicit dynamic analysis. For this purpose, the mass matrix needs to be defined at the very beginning of the calculations using the SHB-EXP elements, while the stiffness matrix is not required in the element formulation. Several methods are available in the literature to compute the mass matrix (see, e.g., Zienkiewicz, 2006). Here, a diagonal lumped element mass matrix $\mathbf{M}^{e}$ is adopted for all SHB-EXP elements. This element mass matrix has a size of $3 n \times 3 n$, with $n$ being the number of nodes in the element, and it is constructed from the following bloc of components:

$$
\mathrm{M}_{I J}=\left\{\begin{array}{lc}
m \int_{\Omega_{e}} \rho N_{I} N_{J} d \Omega & I=J \\
0 & I \neq J
\end{array}, \text { with } \quad m=\int_{\Omega_{e}} \rho d \Omega / \sum_{I=1}^{n} \int_{\Omega_{e}} \rho N_{I} N_{I} d \Omega\right.
$$

where $N_{I}$ and $N_{J}$ are the shape functions, and $\rho$ is the material mass density.

\section{Numerical benchmark tests}

In this section, a representative set of dynamic benchmark tests involving geometric and material non-linearities is selected to assess the performance of the SHB-EXP solid-shell elements. It is worth noting that the converged solutions for these benchmark tests are achieved using only a single element layer with two integration points through the thickness. 
For comparison purposes, all numerical results obtained with the SHB-EXP elements are compared with those given by ABAQUS elements (using similar meshes with the same number of elements in each spatial direction) as well as with reference solutions taken from the literature. The list of SHB-EXP elements with their counterparts from ABAQUS (prismatic and hexahedral elements as well as shell elements) are summarized in Table 2.

Table 2. Prismatic, hexahedral as well as shell finite elements used in the simulations.

In all simulations that follow, the meshes made of hexahedral elements adopt the nomenclature $\mathrm{N}_{1} \times \mathrm{N}_{2} \times \mathrm{N}_{3}$, where $\mathrm{N}_{1}$ indicates the number of elements in the length direction, $\mathrm{N}_{2}$ the number of elements in the width direction, and $\mathrm{N}_{3}$ the number of elements in the thickness direction. For meshes made of prismatic elements, the total number of elements is twice that obtained by using hexahedral elements, due to the in-plane sub-division of a quadrangle into two triangles, resulting in the following nomenclature $\left(\mathrm{N}_{1} \times \mathrm{N}_{2} \times 2\right) \times \mathrm{N}_{3}$. For ABAQUS shell elements, the nomenclature used for triangular shell elements is $\mathrm{N}_{1} \times \mathrm{N}_{2} \times 2$, while the nomenclature for quadrilateral shell elements is simply $\mathrm{N}_{1} \times \mathrm{N}_{2}$. It is worth noting that the solid elements C3D6 and C3D8R in the explicit dynamic code ABAQUS are provided with a single integration point. Therefore, in what follows, several element layers are required for the C3D6 and C3D8R elements in order to have the same number of integration points in the thickness direction as the other elements used for comparison.

\subsection{Cantilever beam bending with a concentrated force}

The elastic cantilever beam bending problem studied by Olovsson et al. (2004) is considered here. The geometric dimensions, material elastic properties, applied loading, and boundary conditions are all specified in Figure 3. For triangular shell or prismatic elements, the meshes include 40 elements uniformly distributed along the length and one element in the width, while for quadrilateral shell or hexahedral elements, the beam is discretized with 10 elements along the length and one element in the width. Figure 4 compares the deflection history at one corner of the free end of the beam obtained with the SHB-EXP elements and ABAQUS elements with the 
reference solution taken from Olovsson et al. (2004). The numerical results show that the mesh used for the C3D6 and C3D8R ABAQUS elements is not sufficient to obtain accurate solutions, due to the poor behavior of these solid elements with regard to locking and hourglassing, while the SHB-EXP elements, as well as the S3R, SC6R, S4R, and SC8R ABAQUS elements are in good agreement with the reference solution. Although requiring twice more elements (i.e., two element layers in the thickness direction), the results given by conventional ABAQUS solid elements are still clearly affected by locking phenomena that are involved in this typical bending-dominated problem, which is not the case of the SHB-EXP elements, thanks to the implementation of the assumed-strain method in their formulation.

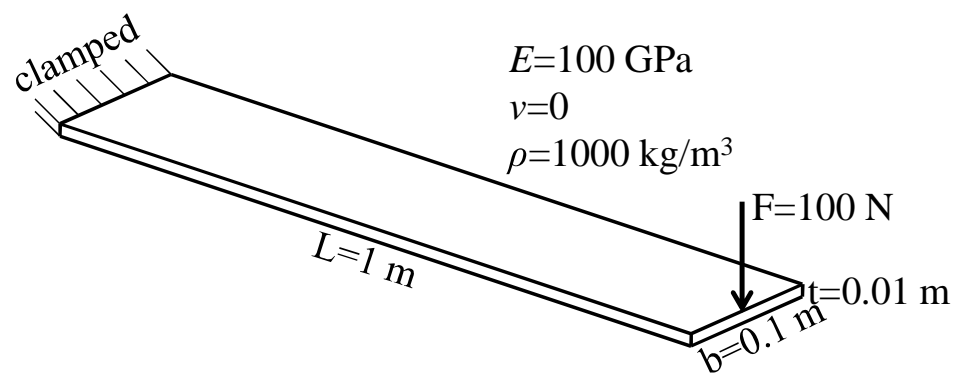

Figure 3. Elastic cantilever beam subjected to a concentrated force

(a)

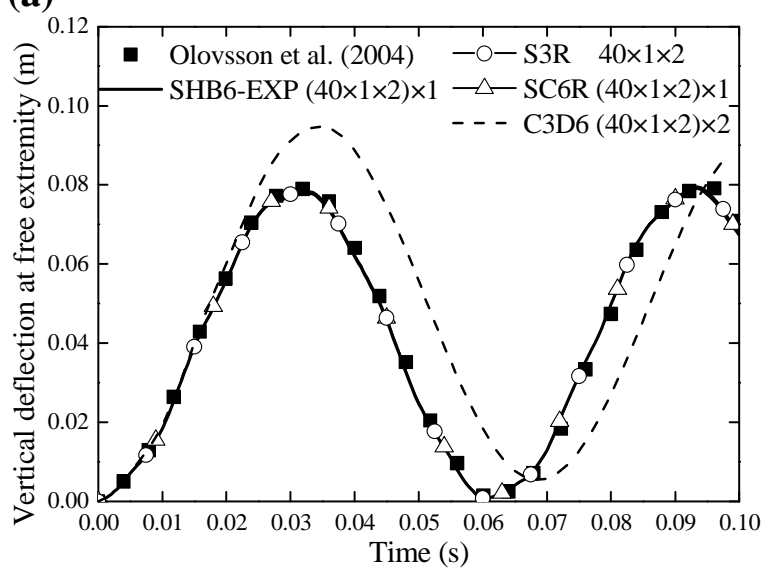

(b)

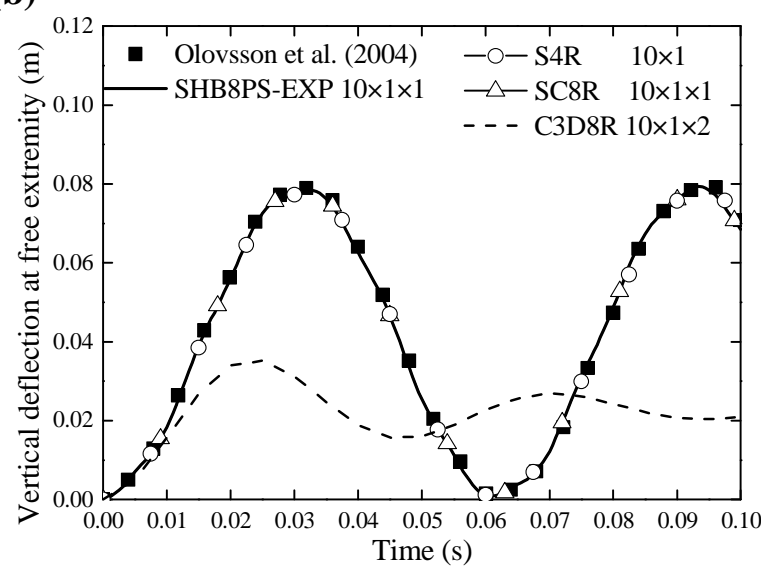

Figure 4. Tip deflection history for the cantilever beam subjected to a concentrated load: using (a) triangular shell / prismatic elements, and (b) using quadrilateral shell / hexahedral elements 


\subsection{Cantilever beam bending with a uniform pressure}

In this subsection, an elastic cantilever beam subjected to a uniform pressure, as proposed by Belytschko et al. (1984), is investigated to assess the bending behavior of the proposed SHB-EXP elements. The geometric dimensions, material elastic properties, applied loading, and boundary conditions are all defined in Figure 5. Finite element simulations have been performed using both the SHB-EXP elements and ABAQUS elements, and the corresponding solutions, in terms of deflection history at one corner of the beam free end (see point A in Figure 5), are compared with the reference solution given in Belytschko et al. (1984). The cantilever beam is discretized with 20 elements uniformly distributed along the length and one element in the width for triangular shell or prismatic elements, and 5 elements along the length and one element in the width for quadrilateral shell or hexahedral elements. Figure 6 depicts all of the numerical results obtained with the SHB-EXP elements and ABAQUS elements. Similar to the previous test, the C3D6 and C3D8R ABAQUS elements require finer meshes to correctly model this cantilever beam bending benchmark problem. Indeed, although necessitating twice more elements (two element layers in the thickness direction), the results displayed by the C3D6 and C3D8R ABAQUS elements still show clear differences with the reference solution, due to their sensitivity to locking effects. By contrast, the results obtained with the proposed SHB-EXP elements show excellent agreement with the reference solution as well as with the S3R, SC6R, S4R, and SC8R ABAQUS elements. It is also revealed, through this bending benchmark test, that the SHB-EXP elements are more suitable to model relatively thick structures than their ABAQUS solid counterparts.

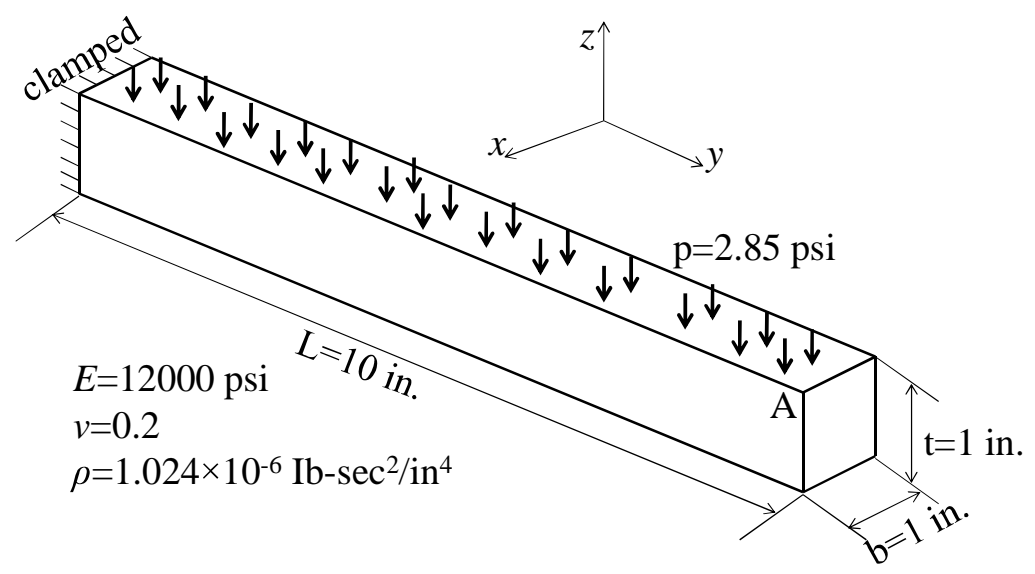

Figure 5. Elastic cantilever beam subjected to a uniform pressure 
(a)

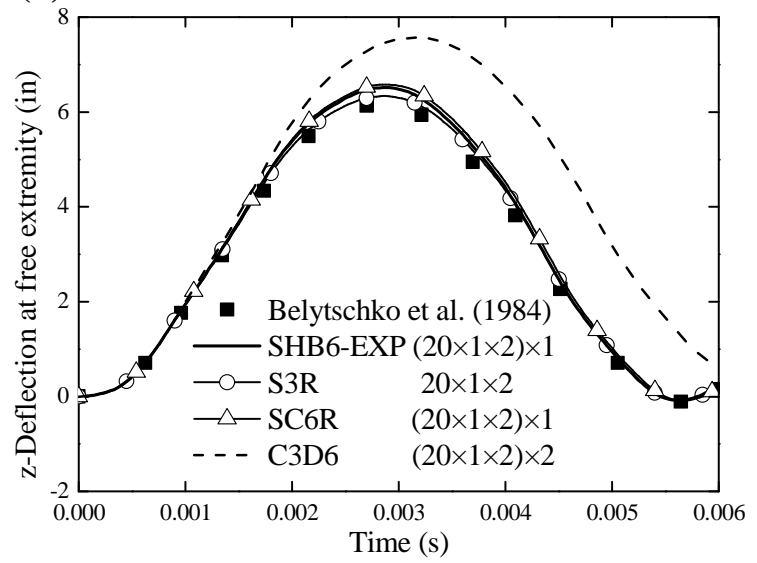

(b)

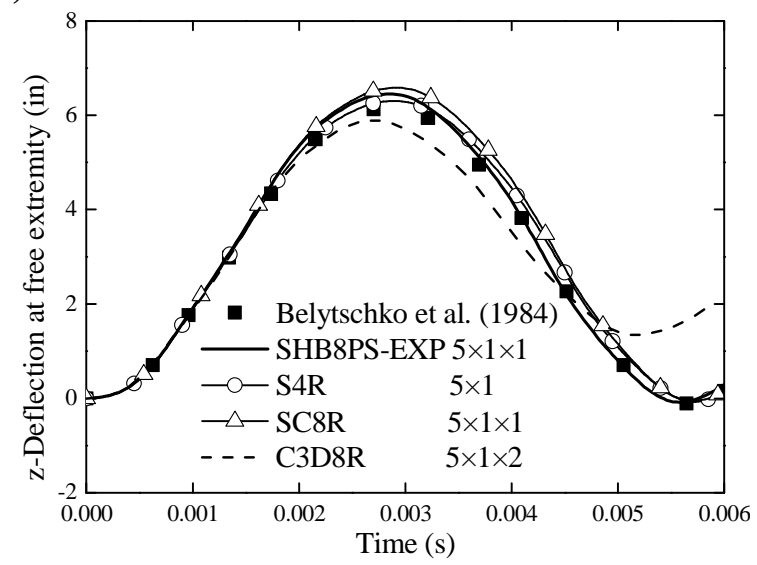

Figure 6. Tip deflection history for the cantilever beam subjected to a uniform pressure: using (a) triangular shell / prismatic elements, and (b) using quadrilateral shell / hexahedral elements

\subsection{In-plane bending of a twisted beam}

The in-plane bending of a twisted beam, as proposed by Belytschko et al. (1992), is a more severe benchmark test than the two previous ones, since it involves both in-plane bending and torsion of the beam. As illustrated in Figure 7, the twisted beam is bent at its free end along the inplane direction. The geometry, material elastic properties and boundary conditions are all specified in Figure 7. Figure 8 compares the deflection history in the load direction at one corner of the free end of the beam, as obtained with the SHB-EXP elements and ABAQUS elements, with the reference solution taken from Belytschko et al. (1992). It can be seen that the SHB-EXP elements perform very well with respect to the reference solution, for this in-plane bending problem, which is also the case for the ABAQUS prismatic solid-shell element SC6R and ABAQUS shell elements. However, as pointed out in the two previous benchmark tests, a finer mesh is required for the ABAQUS solid elements C3D6 and C3D8R, in order to obtain an accurate solution, which is also the case here for the ABAQUS hexahedral solid-shell element SC8R. 


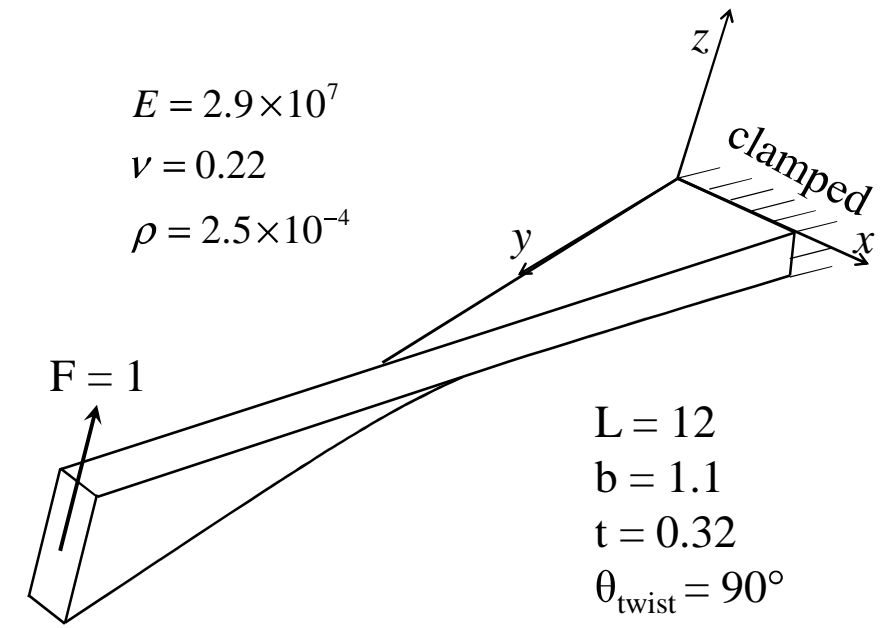

Figure 7. Elastic twisted cantilever beam subjected to in-plane bending load

(a)

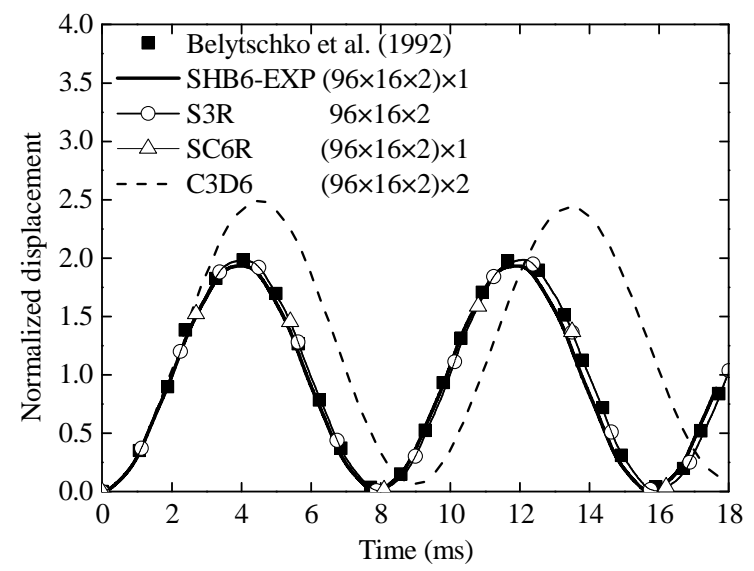

(b)

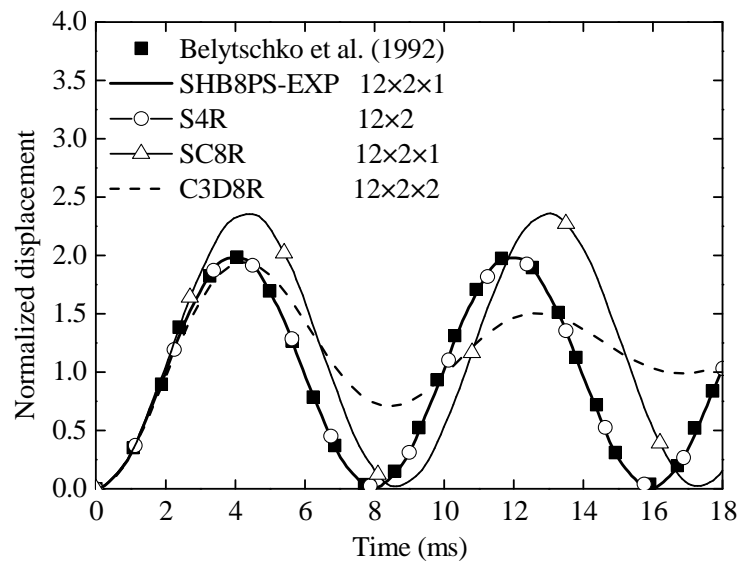

Figure 8. Tip deflection history for the twisted cantilever beam subjected to in-plane bending load: using (a) triangular shell / prismatic elements, and (b) using quadrilateral shell / hexahedral elements

\subsection{Impulsively loaded clamped plate}

A long aluminum plate clamped at both ends is impulsively loaded with a high velocity applied over a central region of the plate surface, as illustrated in Figure 9. The experiment relating to this test has been originally carried out by Balmer and Witmer (1964), and numerically studied subsequently in several literature works (see, e.g., Belytschko et al., 1984; Wu, 2013). The material of the plate is considered as elastic-perfectly-plastic. All details about the geometric 
dimensions, prescribed loading, and elasto-plastic material parameters are summarized in Figure 9. Owing to the symmetry of the problem, one half of the plate is modeled using the proposed SHBEXP elements as well as ABAQUS elements, for comparison purposes.

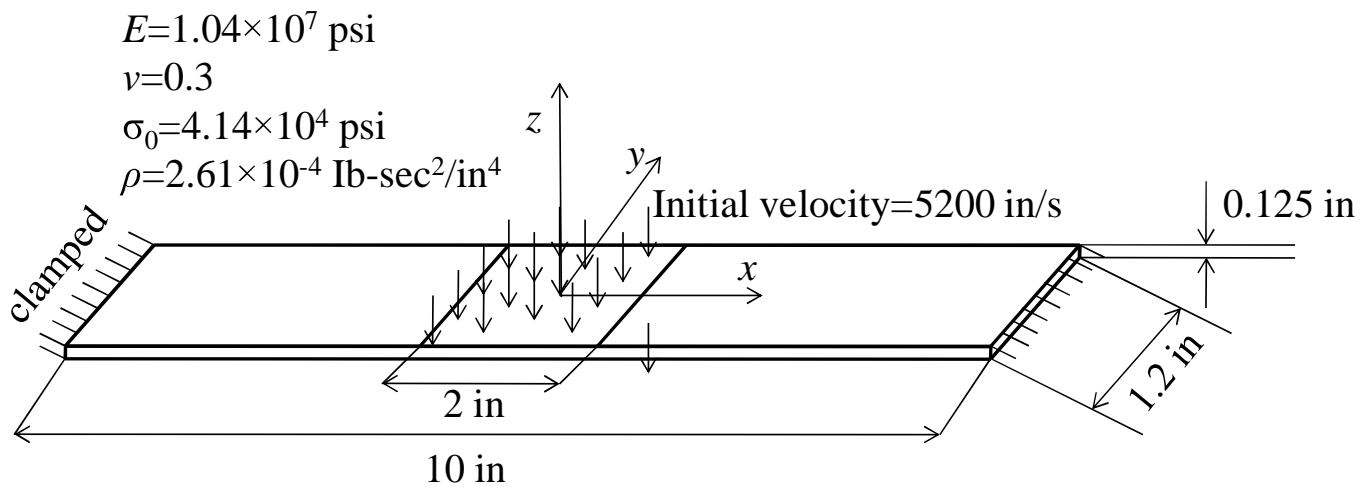

Figure 9. Description of the elastic-plastic clamped plate explosively loaded with an initial velocity.

Figure 10 shows the predictions in terms of the vertical displacement history at the central point of the plate, along with the experimental result taken from Balmer and Witmer (1964) and the numerical reference solution obtained by $\mathrm{Wu}$ (2013). Compared with the simulation results given by ABAQUS elements, the results obtained with the SHB-EXP elements are the closest to the experimental results. This demonstrates the good capabilities of the proposed solid-shell elements in handling elastic-plastic problems with large displacements under highly non-linear dynamic loading conditions. 
(a)

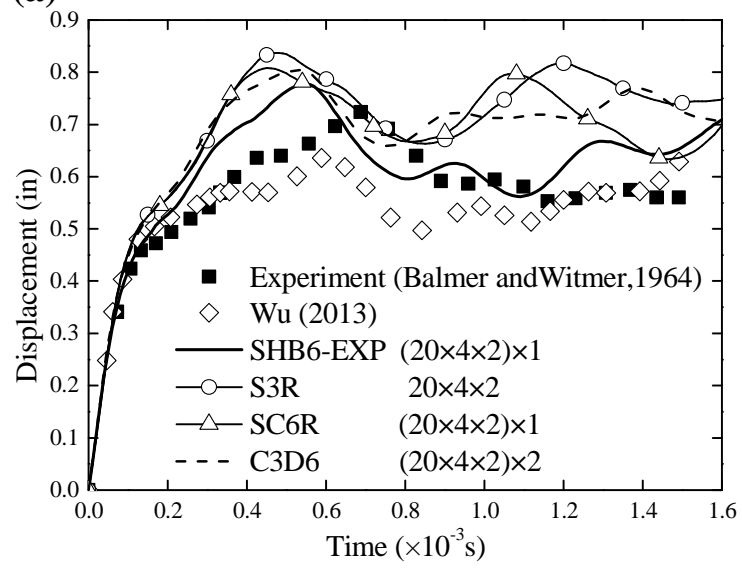

(b)

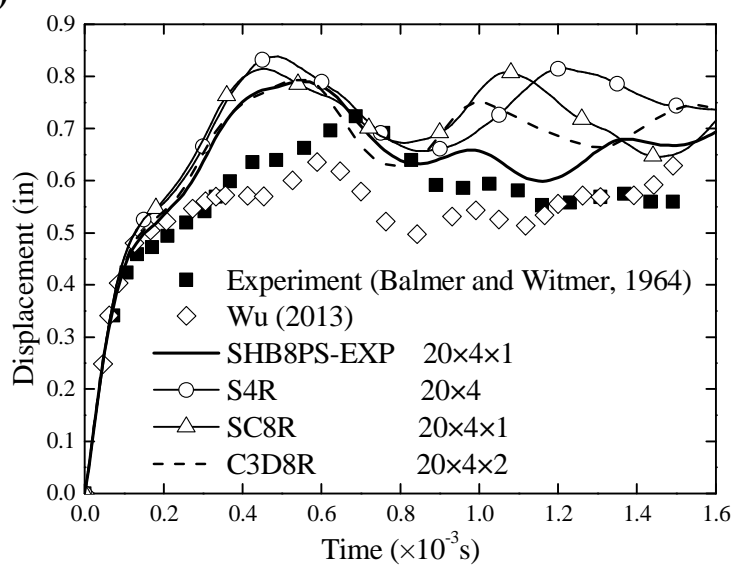

Figure 10. Displacement history at the central point of the elastic-plastic clamped plate: using (a) triangular shell / prismatic elements, and (b) using quadrilateral shell / hexahedral elements

\subsection{Low velocity impact of a circular plate}

The dynamic response of a clamped circular plate subjected to impact by a projectile is investigated here to assess the capabilities of the proposed SHB-EXP elements in dealing with elastic-plastic impact-contact problems. Note that this dynamic benchmark test has previously been studied by Chen et al. (2007) and Mars et al. (2015). The geometric dimensions for the plate and the projectile as well as the prescribed loading and boundary conditions are all defined in Figure 11. The material of the circular plate is made of 6061-T6 aluminum alloy with the following elastic-plastic properties: Young's modulus $E=69 \mathrm{GPa}$, Poisson's ratio $v=0.3$, initial yield stress $\sigma_{0}=290 \mathrm{MPa}$, and mass density $\rho=2600 \mathrm{~kg} / \mathrm{m}^{3}$. The projectile is modeled as a rigid body with an assigned mass at its reference point. The contact between the circular plate and the projectile is assumed to be frictionless, using the hard contact approach available in the ABAQUS/Explicit code. Two typical cases, with different initial weight and velocity for the projectile, are considered:

Case 1: $\mathrm{M}=23.5 \mathrm{~g}, \mathrm{~V}_{0}=49.1 \mathrm{~m} / \mathrm{s}$.

Case 2: $\mathrm{M}=54.4 \mathrm{~g}, \mathrm{~V}_{0}=29.9 \mathrm{~m} / \mathrm{s}$. 


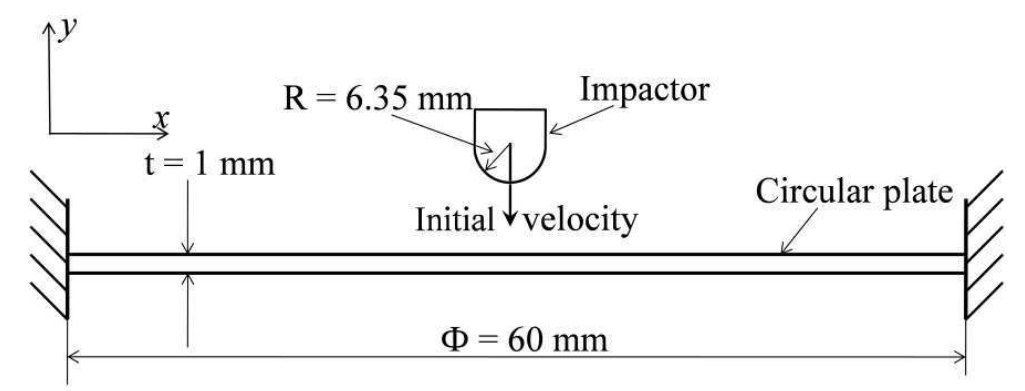

Figure 11. Schematic representation of a circular plate subjected to impact by a projectile

Considering the symmetry of the problem, only one quarter of the plate is modeled using an inplane discretization of 2708 elements in the case of prismatic elements, 2722 elements in the case of triangular shell elements, 934 elements in the case of hexahedral elements, and 951 elements in the case of quadrilateral shell elements (see Figure 12 for illustration). Similar to the previous test, all simulations are carried out using two integration points in the thickness direction, which means two element layers in the case of the ABAQUS C3D8R and C3D6 solid elements, and only a single element layer for all other elements used for comparison. The history of velocity and impact force for the projectile as well as the displacement response at the center of the plate are analyzed using the proposed SHB-EXP elements, which are then compared both with ABAQUS elements and with reference solutions given in Chen et al. (2007). In addition to these numerical comparisons, all of the simulation results are qualitatively compared with the experiments performed by Chen et al. (2007).

(a)

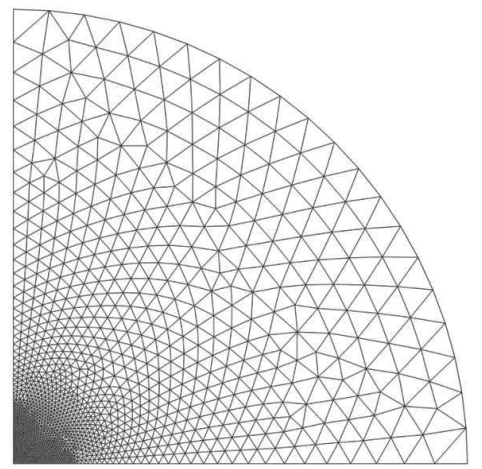

(b)

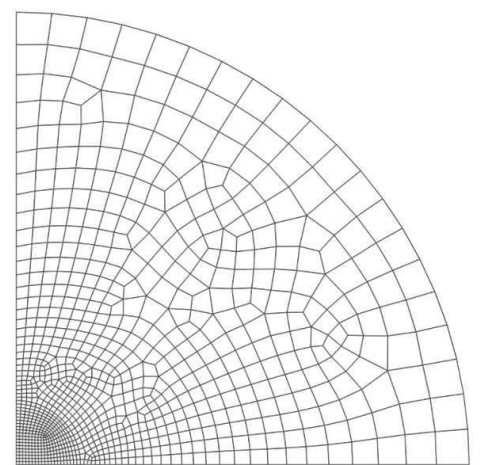

Figure 12. Initial in-plane mesh for the clamped circular plate under impact by a projectile: using (a) triangular shell / prismatic elements, and (b) using quadrilateral shell / hexahedral elements 
Figure 13 shows the experimental results in terms of history of velocity and impact force for the projectile for both studied cases, as reported in Chen et al. (2007). These experimental data help understand the main stages that characterize such impact processes. The first stage corresponds to the elastic-plastic indentation of the circular plate, and lasts until a peak in the impact force response appears. Then, the recovery stage for the circular plate starts, which is characterized by a gradual reduction in the contact between the projectile and the circular plate, until the impact force vanishes. The final stage corresponds to the complete separation between the projectile and the circular plate, which is indicated by a zero impact force and a constant velocity for the projectile.

(a)

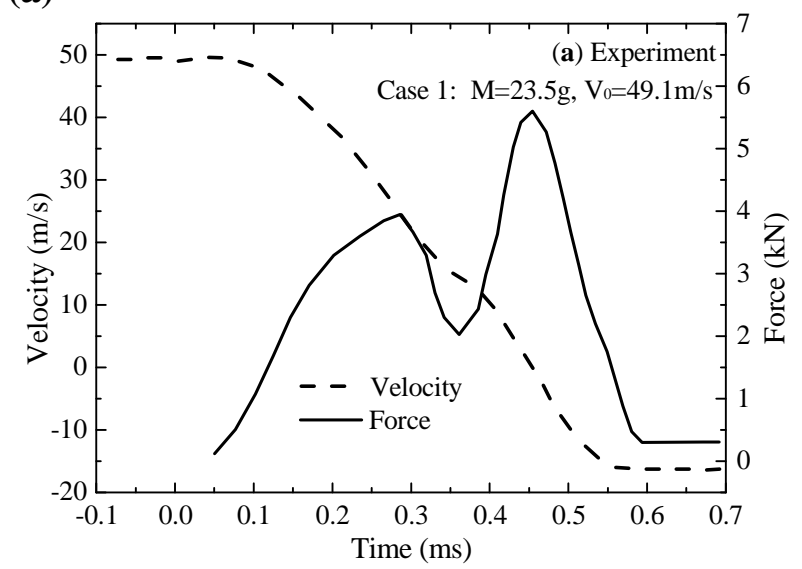

(b)

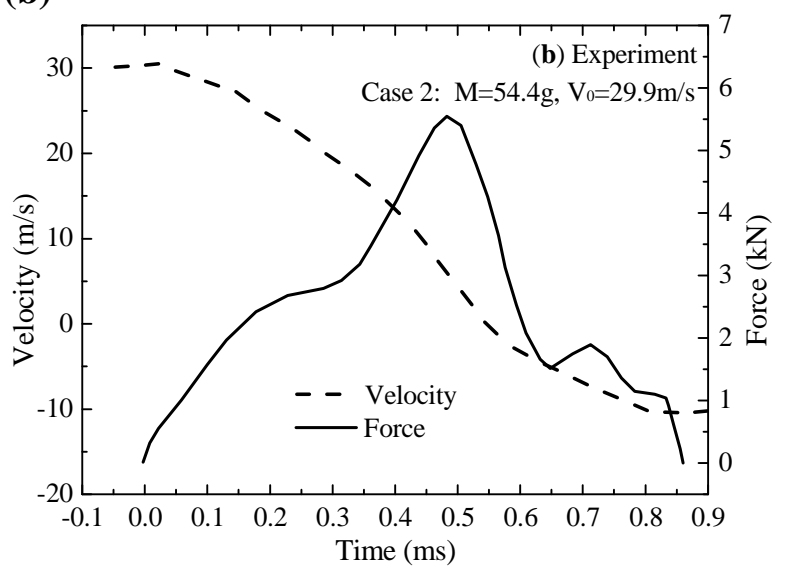

Figure 13. Experimental results in terms of history of velocity and impact force for the projectile for both studied cases: (a) case 1, and (2) case 2

The velocity and the impact force obtained with the SHB-EXP solid-shell elements, for both cases 1 and 2, are compared in Figures 14-19 with the results given by ABAQUS elements as well as with the numerical reference solutions given in Chen et al. (2007). For more clarity in these cross comparisons, the results given by the SHB-EXP elements are compared in Figures 14 and 15 with those yielded by ABAQUS shell elements, in Figures 16 and 17 with ABAQUS solid-shell elements, and in Figures 18 and 19 with ABAQUS solid elements. It can be seen that the double impact force peak, which is typically observed in experiments (see Figures 13(a)-(b)), is well reproduced by the SHB-EXP elements for both studied cases. More specifically, the maximum impact force peak, corresponding to the end of the indentation stage, is reached for the SHB-EXP elements when the velocity of the projectile decreases to zero, which is consistent with the 
numerical reference solutions given by Chen et al. (2007) and the experimental observations in both studied cases.

In terms of comparison with ABAQUS, the results obtained with the SHB-EXP elements show good agreement with both solid and solid-shell ABAQUS elements (see Figures 16-19), while ABAQUS shell elements provide the farthest results with respect to the reference solution. It should be recalled once again that a finer mesh is required for the ABAQUS solid elements, with two element layers in the thickness direction, which involves twice more elements than their solid-shell counterparts.
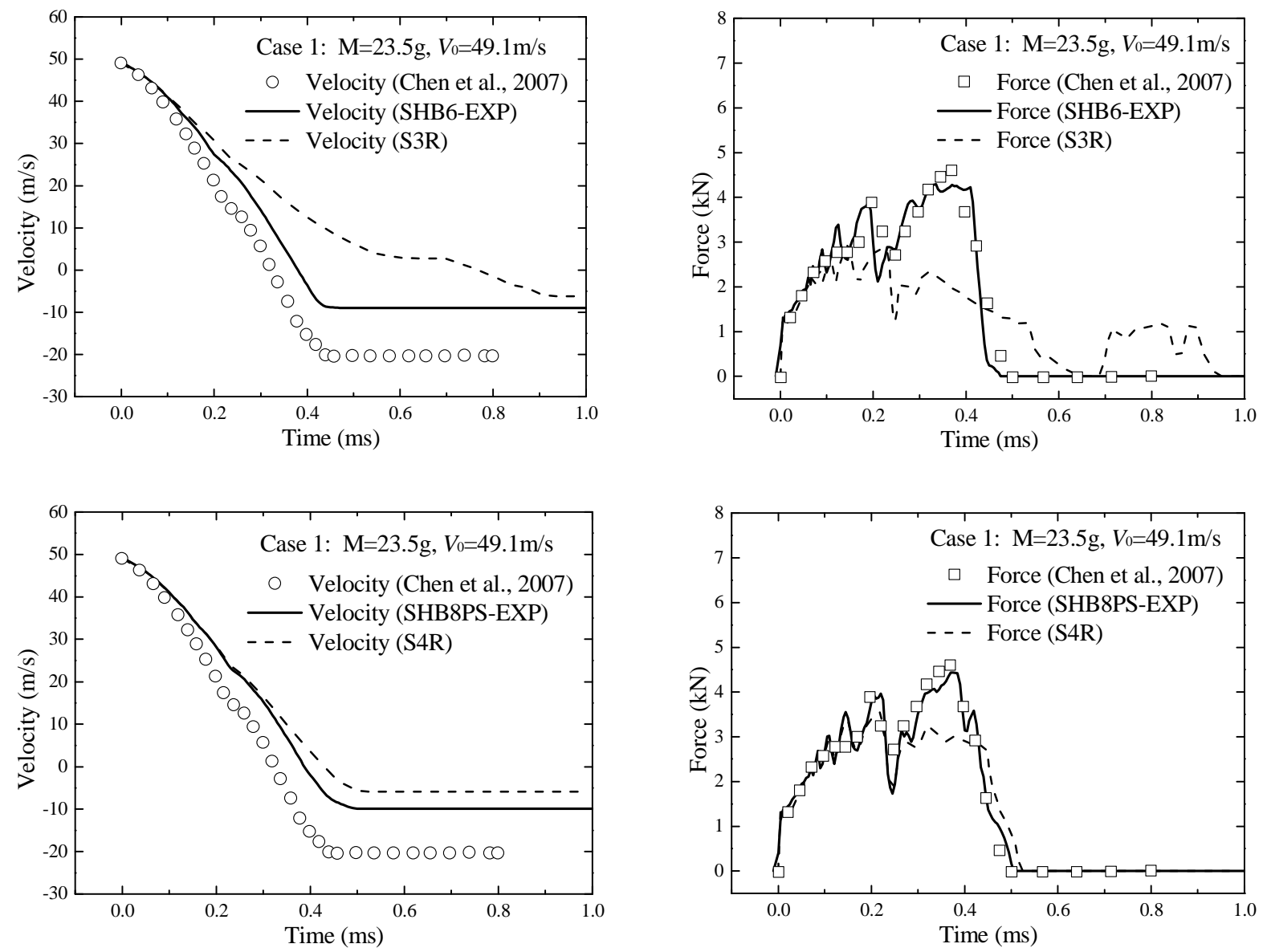

Figure 14. History of velocity (left) and impact force (right) for the projectile, obtained with the SHB-EXP and ABAQUS shell elements along with the reference solutions for case 1 

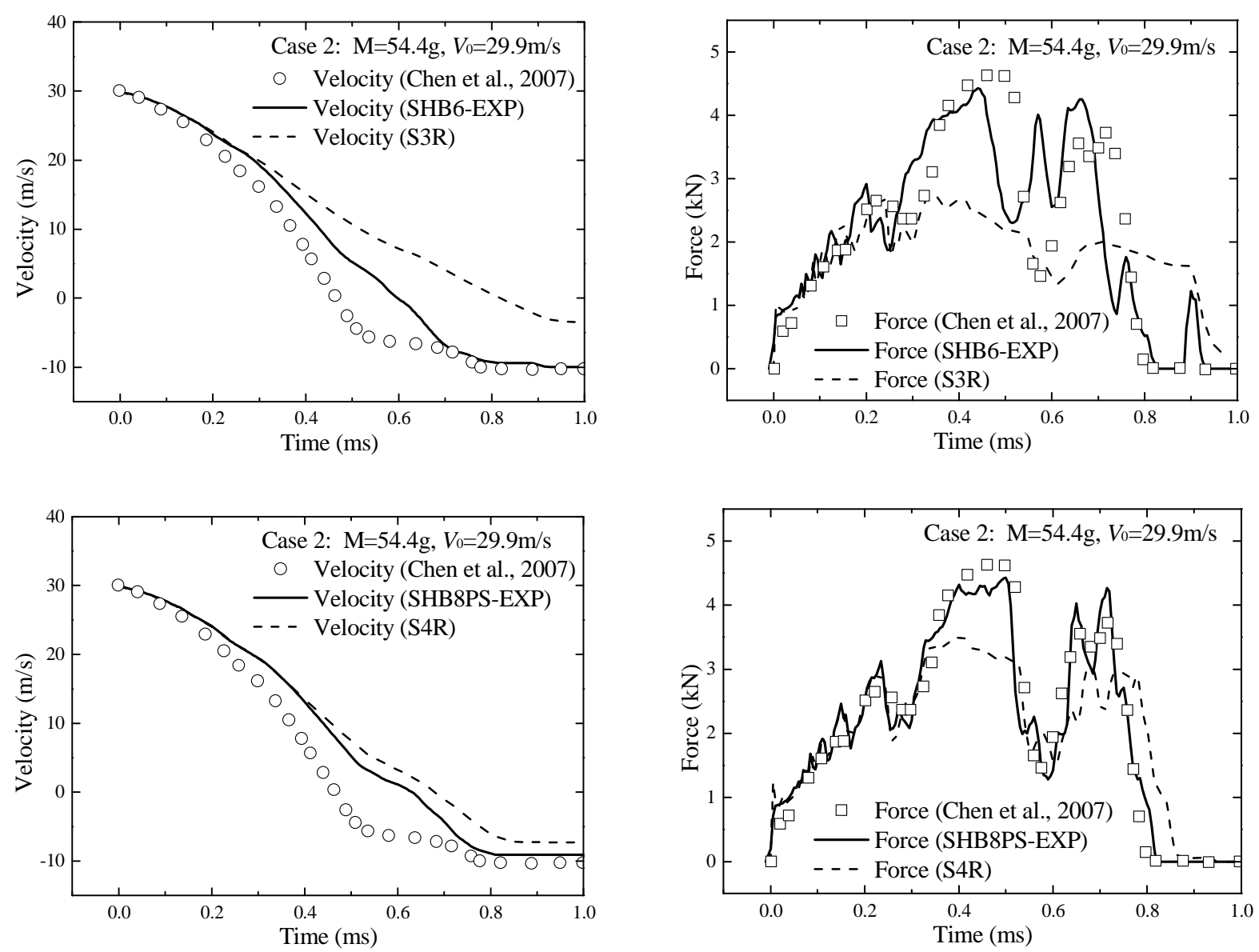

Figure 15. History of velocity (left) and impact force (right) for the projectile, obtained with the SHB-EXP and ABAQUS shell elements along with the reference solutions for case 2 

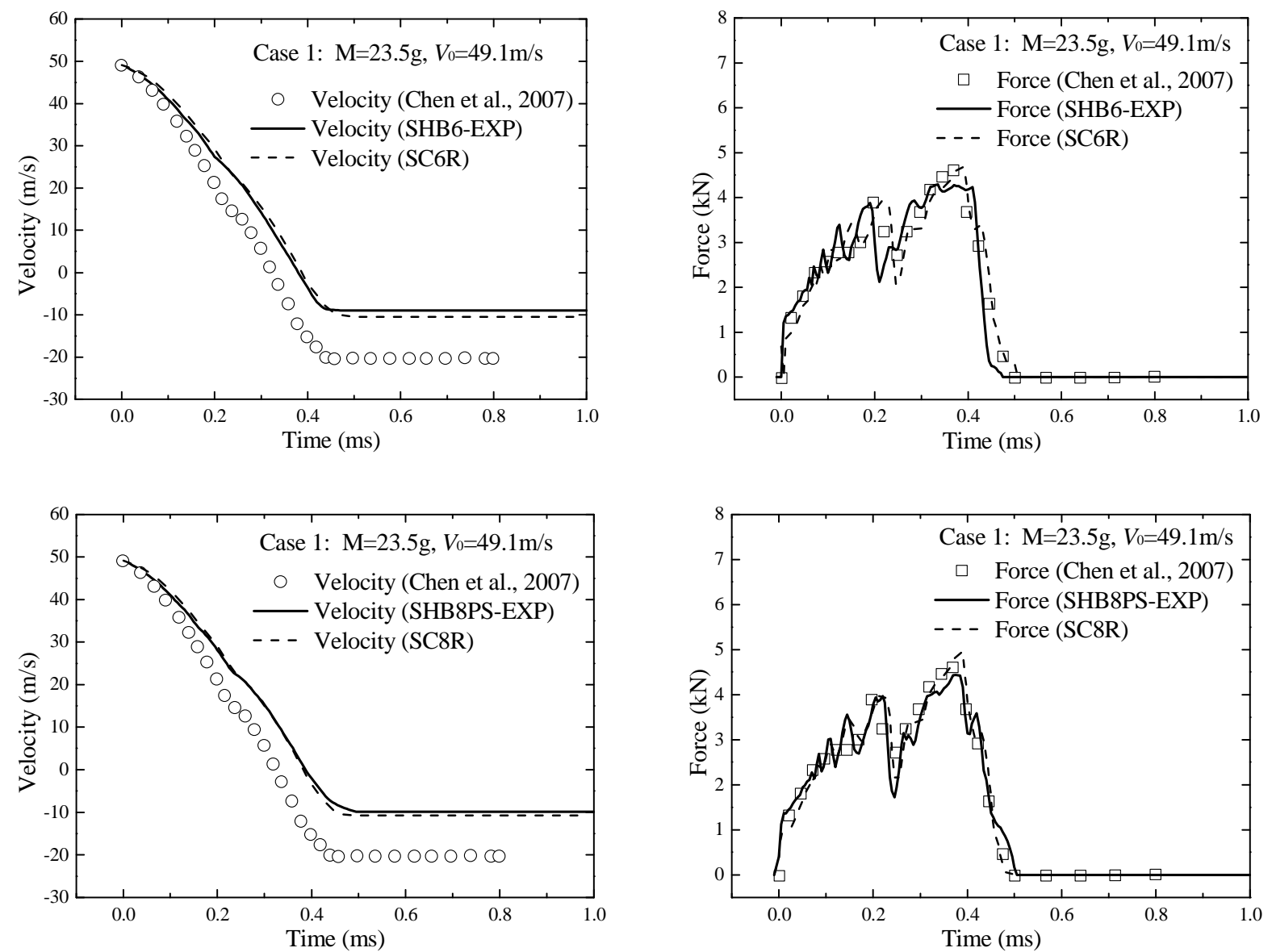

Figure 16. History of velocity (left) and impact force (right) for the projectile, obtained with the SHB-EXP and ABAQUS solid-shell elements along with the reference solutions for case 1 

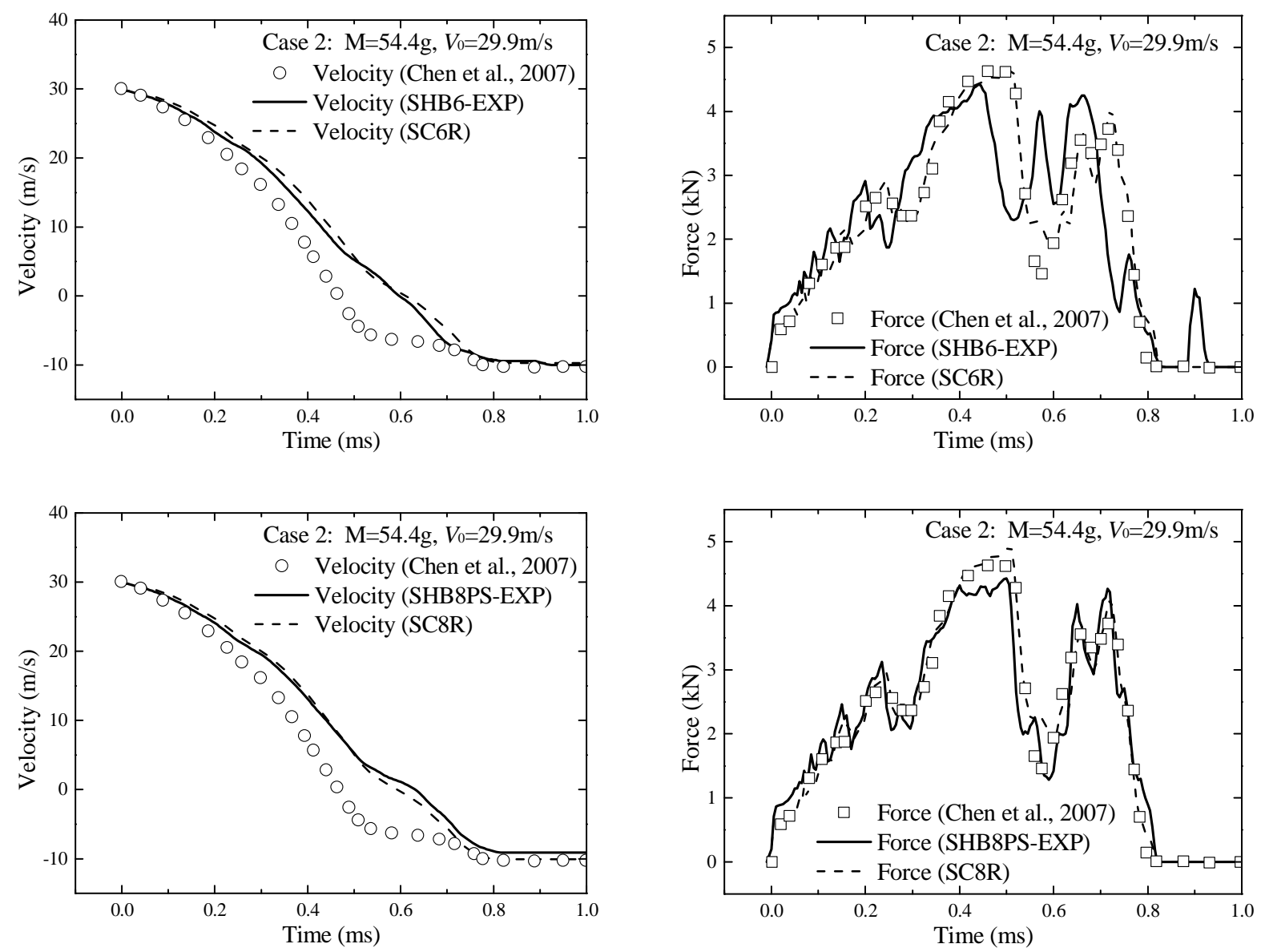

Figure 17. History of velocity (left) and impact force (right) for the projectile, obtained with the SHB-EXP and ABAQUS solid-shell elements along with the reference solutions for case 2 

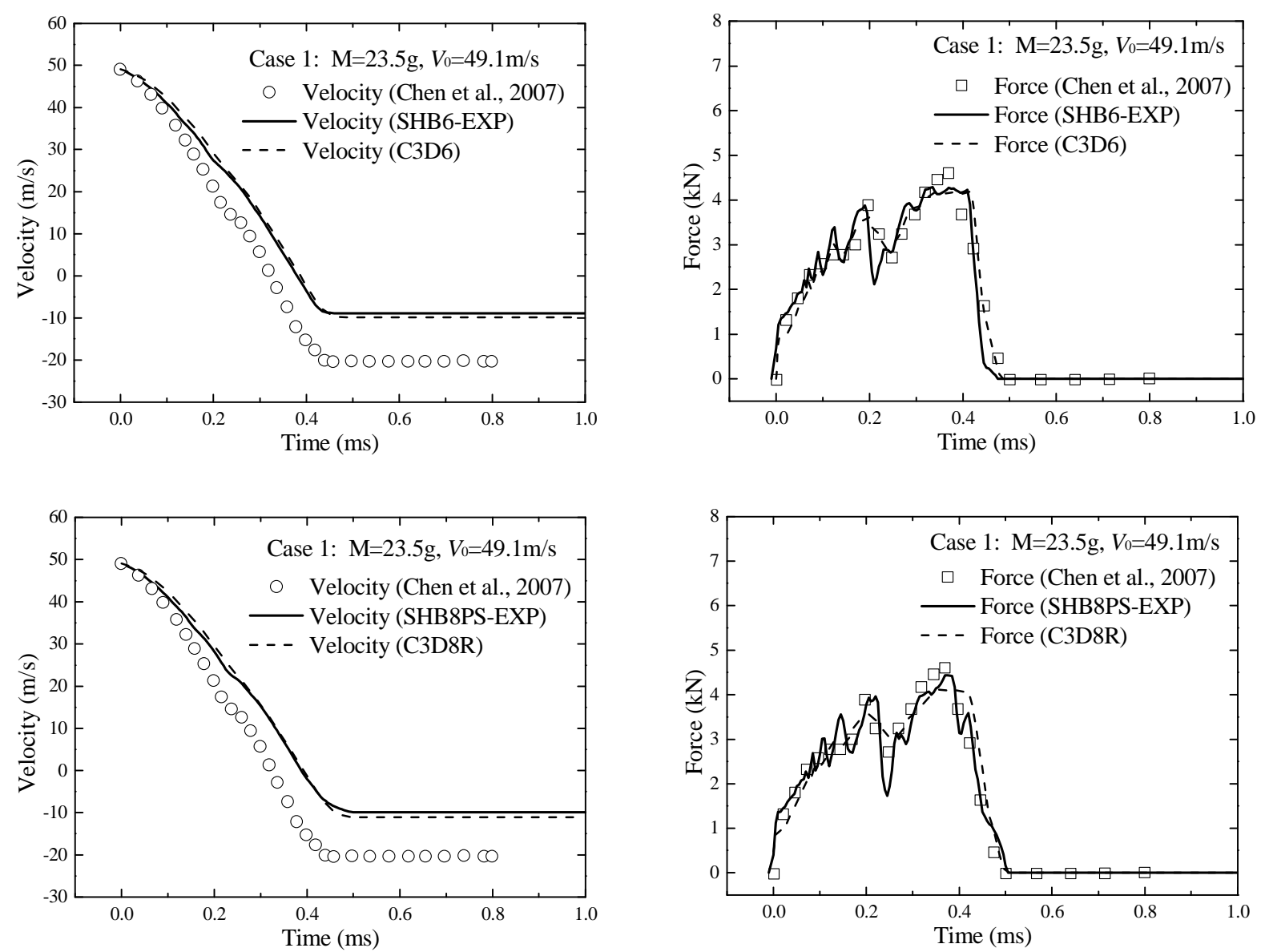

Figure 18. History of velocity (left) and impact force (right) for the projectile, obtained with the SHB-EXP and ABAQUS solid elements along with the reference solutions for case 1 

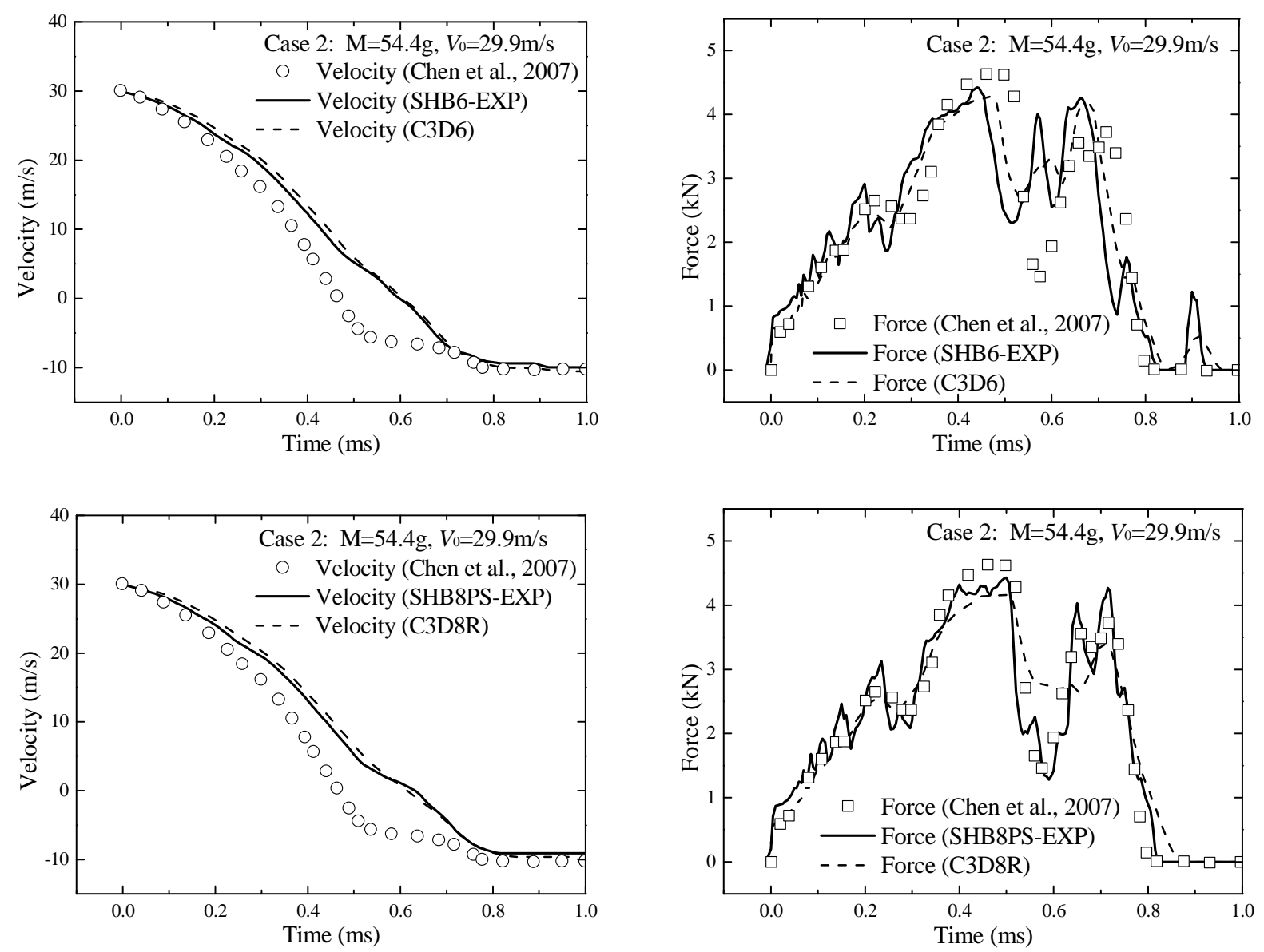

Figure 19. History of velocity (left) and impact force (right) for the projectile, obtained with the SHB-EXP and ABAQUS solid elements along with the reference solutions for case 2

Furthermore, the displacement history at the plate center is also investigated. For this purpose, Figure 20 compares the displacements obtained with the SHB-EXP elements, for both studied cases, with those yielded by ABAQUS elements as well as with the numerical reference solutions given by Chen et al. (2007). Note that no experimental measurements for the center plate displacement have been reported in the literature. Overall, it can be observed that the SHB-EXP elements provide the closest results, with respect to the reference solutions, in comparison with ABAQUS solid and solid-shell elements, while the results yielded by ABAQUS shell elements appear to be the farthest. 

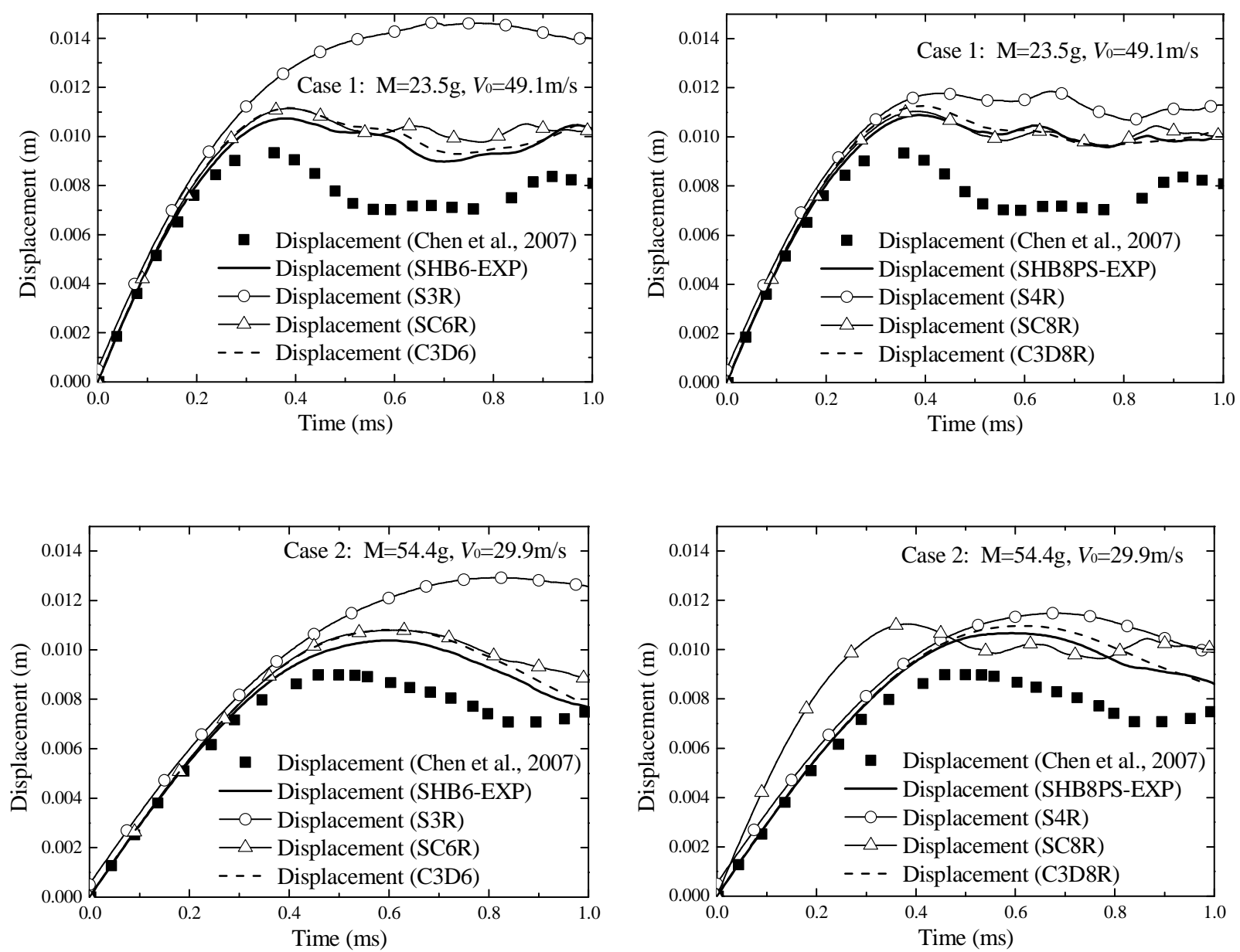

Figure 20. Displacement history at the plate center, obtained with the SHB-EXP and ABAQUS elements along with the reference solutions (Chen et al., 2007), for case 1 (top) and case 2 (bottom)

\subsection{Elastic-plastic ball impacting a circular plate}

In this subsection, another impact benchmark problem, previously studied by Olovsson et al. (2004), is investigated using the proposed SHB-EXP elements. This test consists in a ball, with a high initial velocity of $400 \mathrm{~m} / \mathrm{s}$, impacting a clamped circular plate (see Figure 21). The initial radius of the ball is $R_{b}=60 \mathrm{~mm}$, while the radius and the thickness of the clamped circular plate are $R_{p}=200 \mathrm{~mm}$ and $t_{d}=2 \mathrm{~mm}$, respectively. The ball is modeled as a deformable body with elastic-perfectly-plastic behavior, whereas the circular plate is described by an elastic-plastic model with linear isotropic hardening (see Table 1). The material parameters used in the simulations, for both the ball and the circular plate, are summarized in Table 3. Owing to the symmetry, only one quarter of the ball and the circular plate is modeled. The ball is discretized 
with 1728 C3D8I ABAQUS elements, which consist of 8-node solid elements based on the incompatible mode approach (see, e.g., Taylor et al., 1976; Wilson and Ibrahimbegovic, 1990). In order to obtain a regular mesh for the circular plate quarter, the latter is divided into three regions, each having four edges (see illustration in Figure 22(a)). The mesh nomenclature adopted in the simulations is $3 \times\left(\mathrm{N}_{1} \times \mathrm{N}_{1} \times \mathrm{N}_{3}\right)$ for hexahedral elements, where $\mathrm{N}_{1}$ indicates the number of elements along each edge and $\mathrm{N}_{3}$ the number of elements in the thickness direction (see Figure 22(b)). For prismatic elements, the total number of elements is twice that corresponding to hexahedral elements, which leads to $3 \times\left(\left(\mathrm{N}_{1} \times \mathrm{N}_{1} \times 2\right) \times \mathrm{N}_{3}\right)$ elements (see Figure 22(c)). For quadrilateral shell elements, the nomenclature for discretizing the quarter model is $3 \times\left(\mathrm{N}_{1} \times \mathrm{N}_{1}\right)$, while this nomenclature is $3 \times\left(\mathrm{N}_{1} \times \mathrm{N}_{1} \times 2\right)$ when triangular shell elements are used. For all simulations, the contact between the ball and the circular plate is assumed to be frictionless.

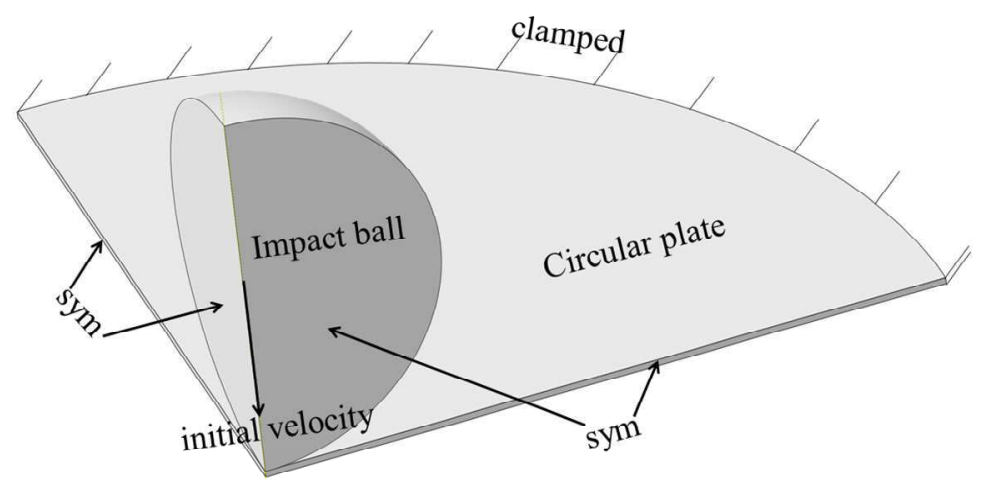

Figure 21. Schematic representation of a ball impacting a clamped circular plate

Table 3. Material parameters for the ball and the circular plate.

Figure 22 shows the initial meshes and final deformed shapes of the circular plate, as obtained with the SHB8PS-EXP and SHB6-EXP elements. In Figure 23, the deflection history at the central point of the plate obtained with the SHB-EXP elements is compared with that yielded by ABAQUS elements as well as with the numerical reference solution given by Olovsson et al. (2004). One can observe that the results obtained with the SHB-EXP elements are comparable in terms of accuracy to those given by ABAQUS elements, and are also in good agreement with the reference solution. 
Note however that the ABAQUS solid elements require two element layers in the thickness direction to provide comparable accuracy, which means twice more elements than their solid-shell counterparts, while the S4R ABAQUS shell element requires a slightly finer mesh.

(a)

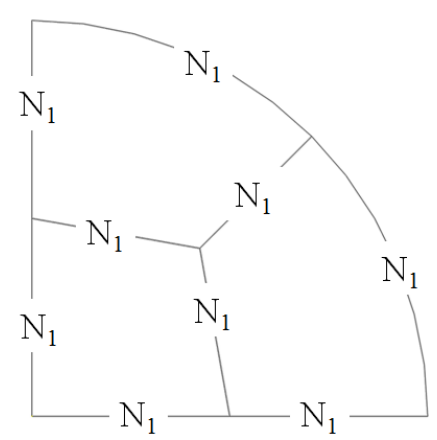

(d) (b)

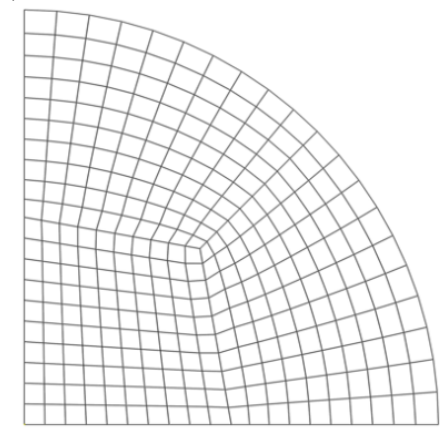

(e) (c)

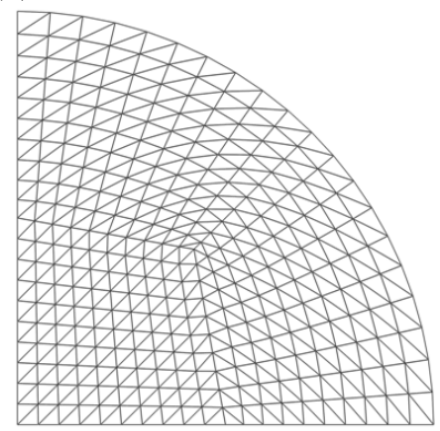

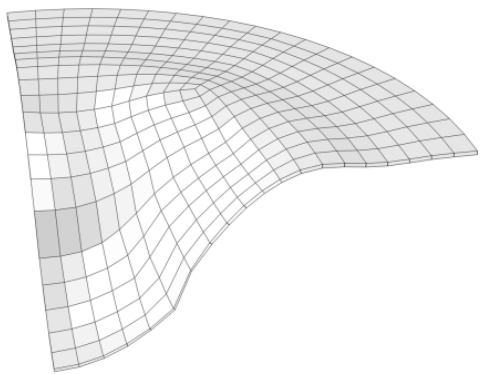

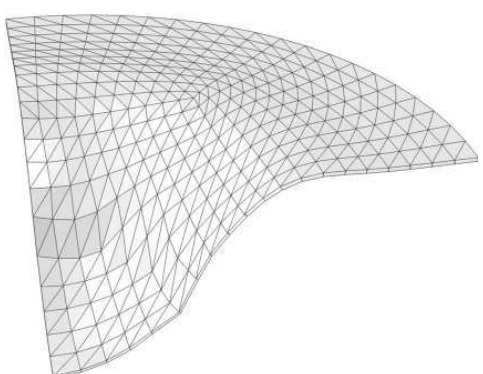

Figure 22. Initial meshes and final deformed shape for the quarter model of the circular plate

(a)

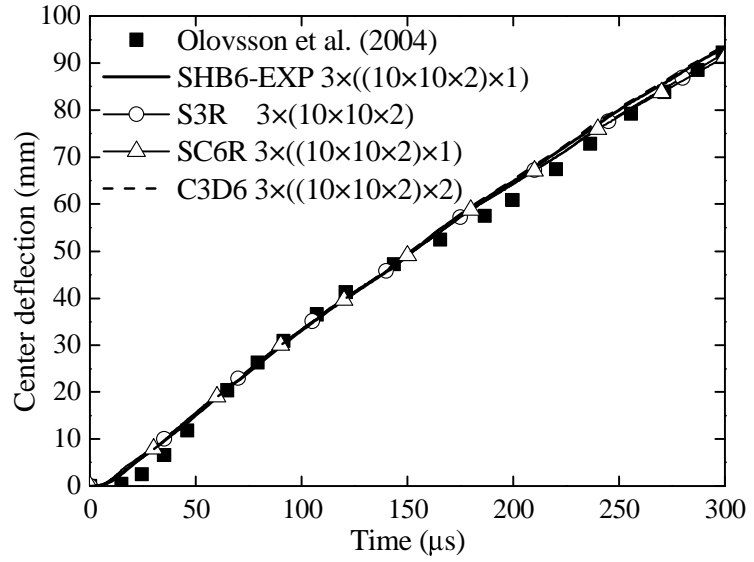

(b)

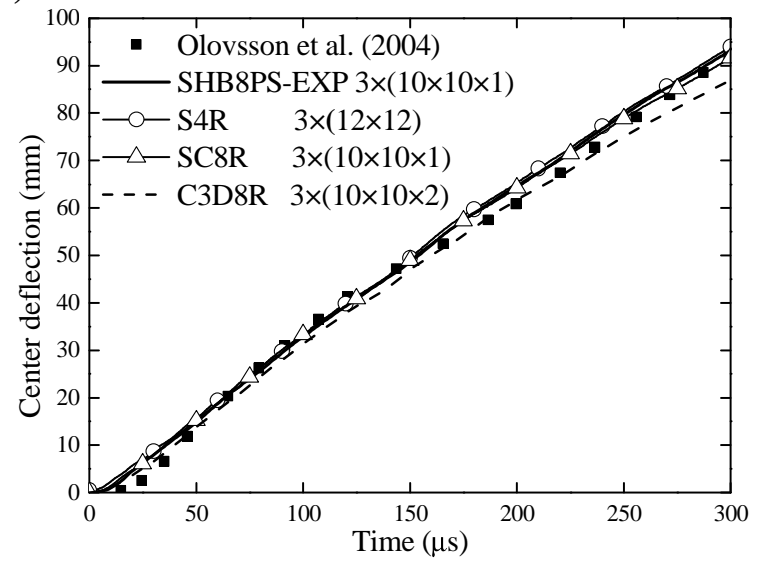

Figure 23. Deflection history at the central point of the clamped circular plate under impact: using (a) triangular shell / prismatic elements, and (b) using quadrilateral shell / hexahedral elements 


\section{Application to the simulation of sheet metal forming processes}

In this section, three deep drawing tests with various punch shapes, involving different materials and associated anisotropy properties, are simulated with the present SHB-EXP elements in order to evaluate their performance in the context of sheet metal forming simulation. The obtained results are compared with those given by ABAQUS elements, on the one hand, and with experimental data taken from the literature, on the other hand.

\subsection{Deep drawing of a square cup}

The deep drawing of a square cup, initially proposed in Numisheet'93 conference (Makinouchi et al., 1993), is considered here to further assess the performance of the SHB-EXP elements in the context of sheet metal forming processes, which involve large plastic deformations, material nonlinearity and anisotropy, and double-sided contact. Note that this benchmark test is very popular within the sheet metal forming community and, accordingly, it has been investigated by a number of authors in the literature (see, e.g., Choi and Huh, 1999; Schwarze et al., 2011; Pagani et al., 2014). The schematic view of the setup and the associated geometric dimensions are shown in Figure 24.

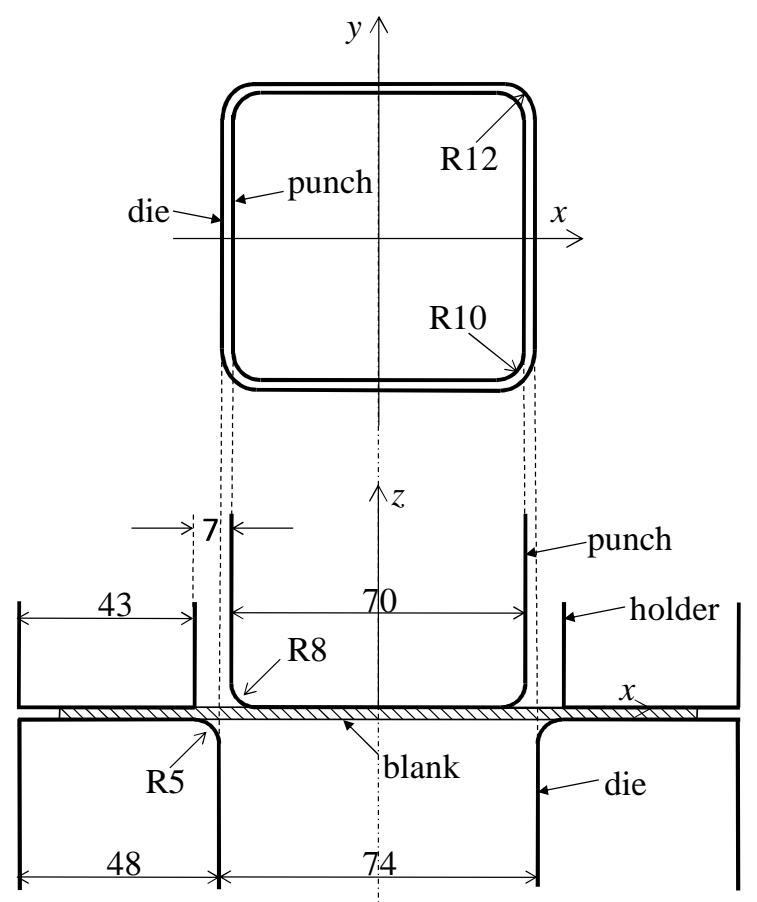

Figure 24. Schematic view for the square cup drawing setup 
The initial dimensions of the sheet are $150 \mathrm{~mm} \times 150 \mathrm{~mm} \times 0.81 \mathrm{~mm}$. The sheet metal is made of an aluminum material whose behavior is described by the Swift isotropic hardening law (see Table 1) along with a von Mises-type yield criterion. The associated material parameters are summarized in Table 4.

Table 4. Elastic-plastic parameters for the studied aluminum alloy.

All along the forming process, a constant blank holder force of $16.6 \mathrm{kN}$ is applied, and the friction coefficient between the forming tools and the sheet is taken equal to 0.162 (Makinouchi et al., 1993). Only one quarter of the problem is discretized, considering the symmetry of the tools and the sheet.

Figure 25 shows the final deformed drawn of the square cup at the punch displacement of 15 $\mathrm{mm}$, as obtained with the prismatic and hexahedral SHB-EXP elements.

(a)

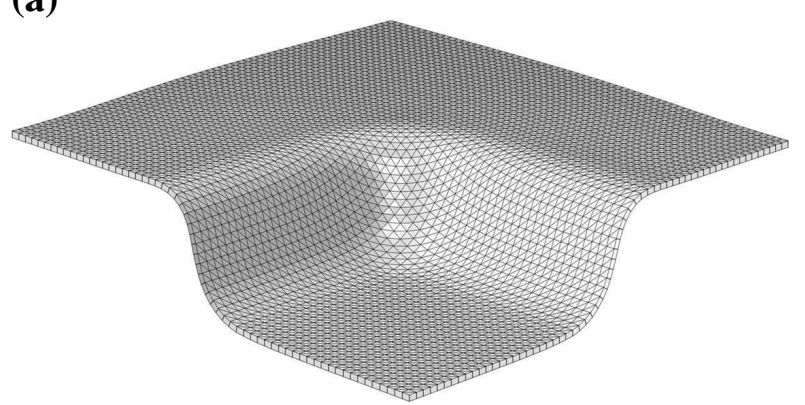

(b)

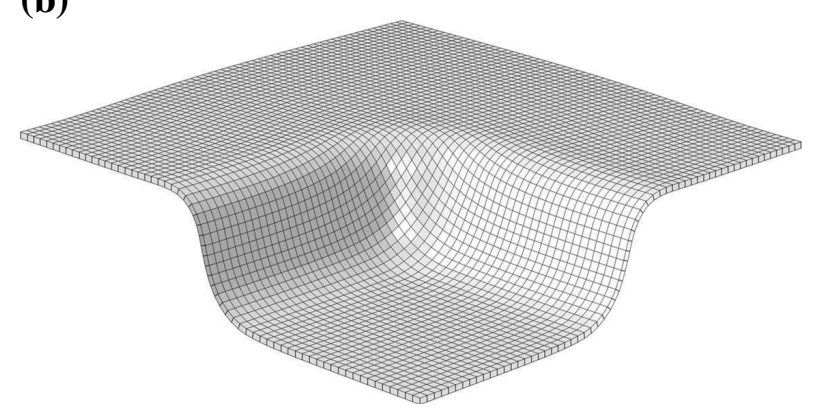

Figure 25. Final deformed drawn of the square cup at a punch displacement of $15 \mathrm{~mm}$ : using (a) SHB6-EXP elements, and (b) using SHB8PS-EXP elements

Three final draw-in distances, as illustrated in Figure 26, are analyzed and compared with the experimental measurements (Makinouchi et al., 1993), where $\mathrm{D}_{\mathrm{x}}$ denotes the draw-in distance along the $\mathrm{x}$-axis, $\mathrm{D}_{\mathrm{y}}$ along the $\mathrm{y}$-axis, and $\mathrm{D}_{\mathrm{d}}$ the draw-in distance along the diagonal direction. The in-plane discretization of the square sheet uses $64 \times 64$ elements, in the case of quadrilateral shell or hexahedral elements, and $64 \times 64 \times 2$ elements, in the case of triangular shell or prismatic elements. The sensitivity of the simulation results to the number of through-thickness Gauss 
integration points (GIP) is also analyzed. For the SHB-EXP elements (SHB6-EXP and SHB8PSEXP), ABAQUS shell elements (S3R and S4R), and ABAQUS solid-shell elements (SC6R), the simulations are carried out using 2,3, and 5 GIP points, successively, with only a single element layer. However, for ABAQUS solid elements (C3D6 and C3D8R), several element layers are used to mesh the sheet thickness (i.e., 2, 3 and 5 layers), in order to have a comparable number of GIP. Note that the SC8R ABAQUS solid-shell element failed to simulate the present benchmark test and, therefore, no predictions are presented for this element.

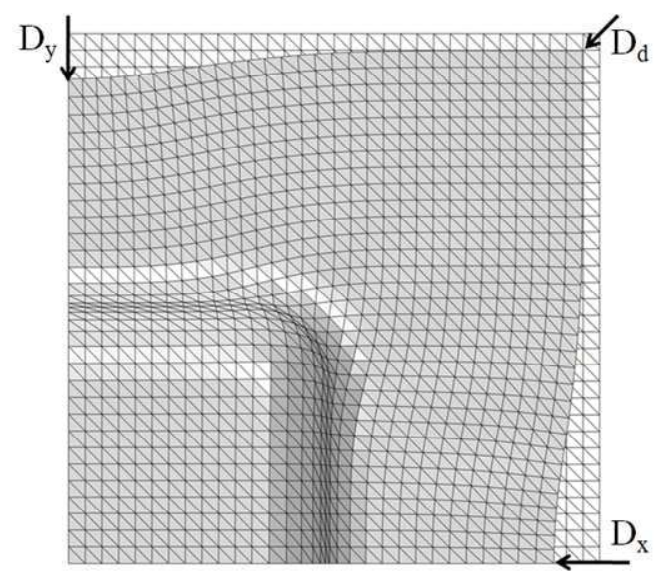

Figure 26. Illustration of the draw-in distances of the square cup after forming

Figure 27 compares the draw-in distances predicted by the SHB-EXP elements and ABAQUS elements, for different through-thickness GIP, with the experimental measurements from Makinouchi et al. (1993). A first observation is that most of the simulation results lie in the range delimited by the minimum and the maximum draw-in experimental measurements.

For the SHB6-EXP prismatic solid-shell element, the simulation results reported in Figures 27(a), (c) and (e) suggest that at least 3 through-thickness GIP should be used to obtain accurate predictions for this forming process. When this condition is met, the SHB6-EXP element provides results that fall in the range delimited by the minimum and the maximum draw-in experimental measurements, and which are the closest to the average draw-in experimental distances. It is worth noting that the $D_{x}$ and $D_{y}$ draw-in distances predicted with the S3R ABAQUS shell element are overestimated, showing limitations of shell elements in modeling sheet metal forming processes involving double-sided contact. 
For the SHB8PS-EXP hexahedral solid-shell element (see Figures 27(b), (d) and (f)), the associated simulation results are comparable to those given by the C3D8R ABAQUS solid element; however, the latter requires resorting to several element layers in contrast to the proposed solid-shell elements. On the other hand, the predictions using the S4R ABAQUS shell element are overestimated in most cases, revealing once again the limitations of shell elements in handling double-sided contact. Finally, the sensitivity study to the number of through-thickness GIP reveals that only two GIP are sufficient for the SHB8PS-EXP element to accurately describe the various non-linear through-thickness phenomena. 
(a)

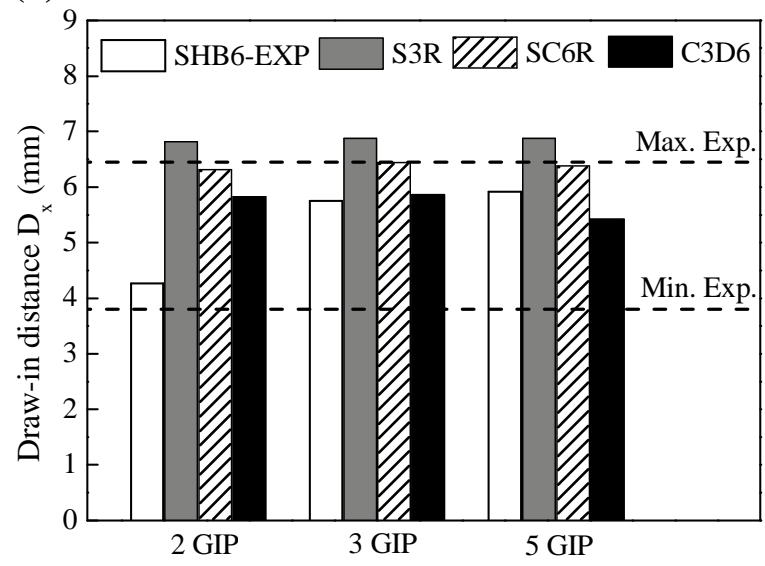

(c)

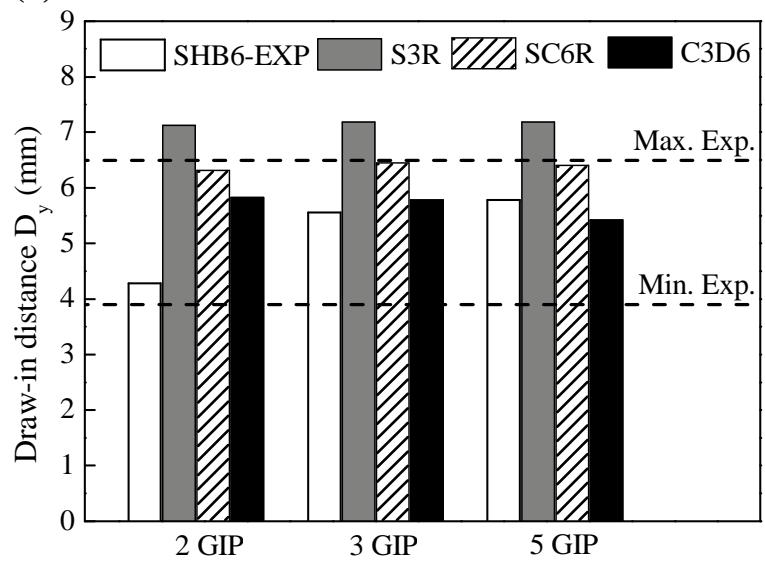

(e)

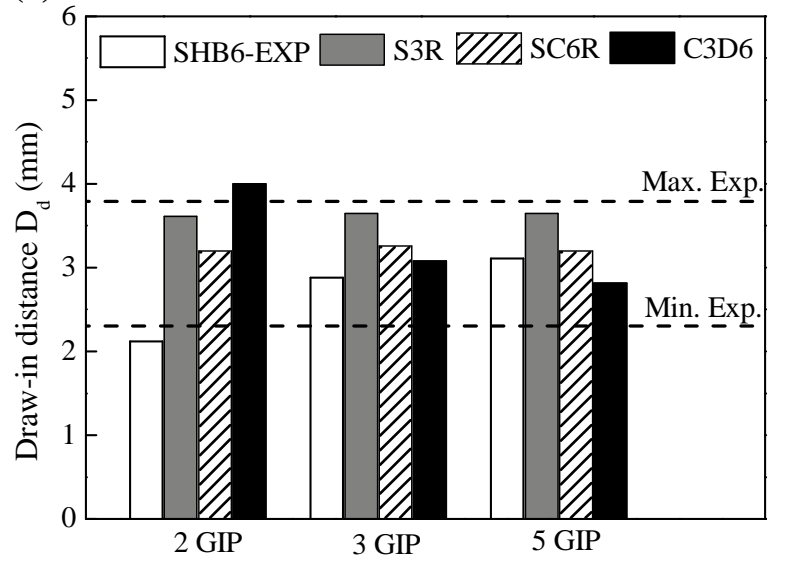

(b)

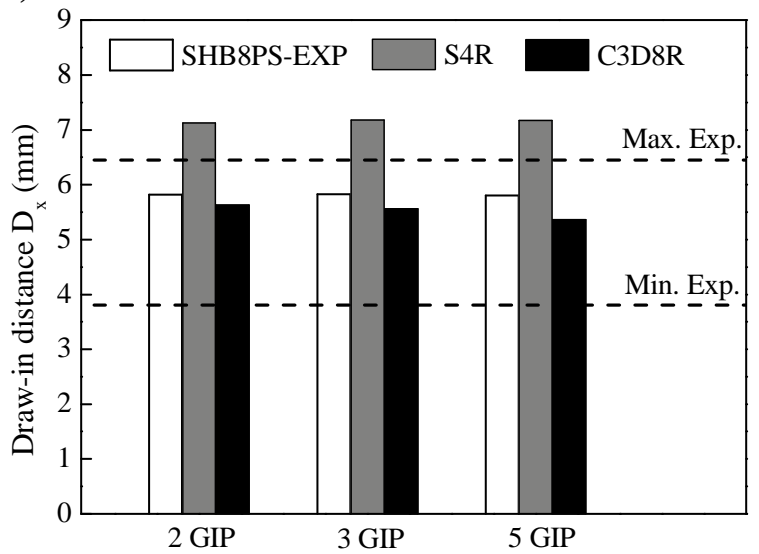

(d)

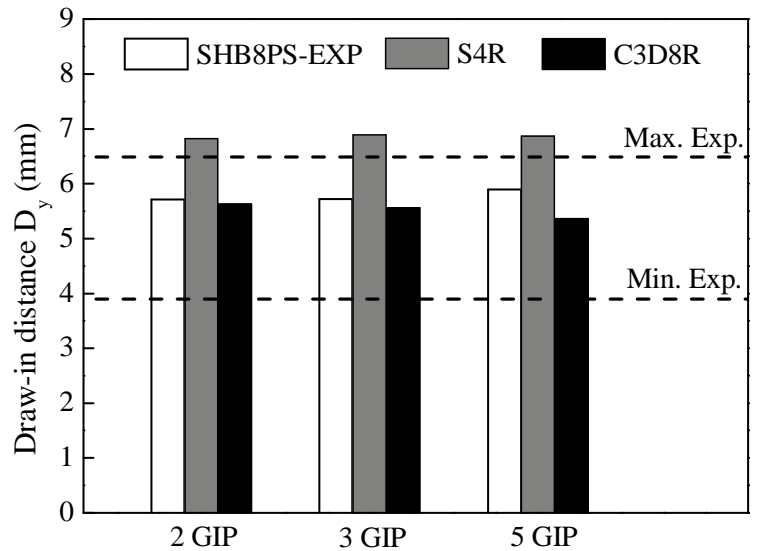

(f)

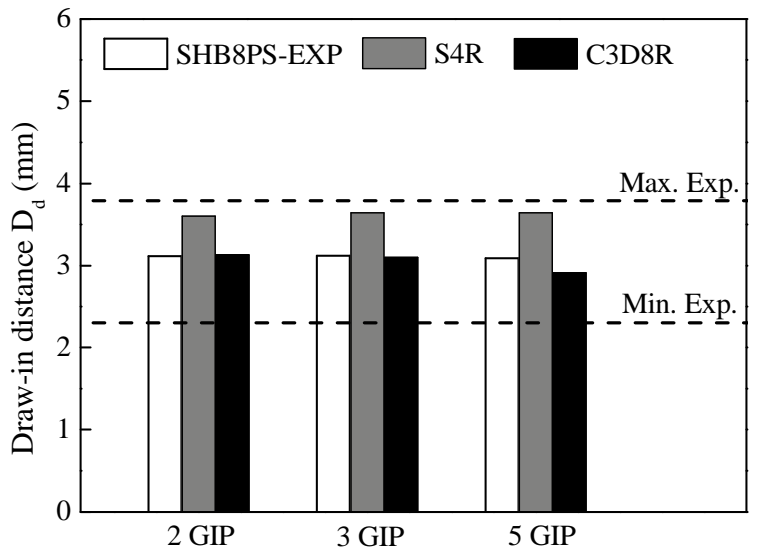

Figure 27. Comparison of the draw-in distances at $15 \mathrm{~mm}$ punch displacement, as obtained by the SHB-EXP elements, ABAQUS elements and experimental measurements 


\subsection{Deep drawing of a rectangular cup}

The second sheet metal forming test investigated in this work consists in the deep drawing of a rectangular sheet with tools of rectangular shape. Because of its particular geometric characteristics, this benchmark problem is known to involve strong non-linearities (due to large deformations, plasticity, and contact), which are more severe than those encountered in the deep drawing of a square cup (see subsection 4.1). This test has been previously studied by Choi and Huh (1999) and Huh and Choi (1999), by considering the Hill'48 quadratic yield criterion for the plastic anisotropy of the sheet and the Swift law for isotropic hardening. The initial dimensions of the rectangular sheet are $120 \mathrm{~mm} \times 170 \mathrm{~mm} \times 0.625 \mathrm{~mm}$. The material parameters of the sheet metal, corresponding to a cold rolled steel, are summarized in Table 5 (see Choi and Huh, 1999), while the details on the geometry of the forming setup and its dimensions are all reported in Figure 28.

Table 5. Material parameters associated with the anisotropic elastic-plastic model for the cold rolled steel.

The constant blank holder force is set equal to $14.71 \mathrm{kN}$ during the forming process, and the Coulomb friction coefficient between the forming tools and the sheet is taken equal to 0.11 . Owing to the symmetry, only one quarter of the sheet is modeled, with an in-plane discretization of 1960 elements for triangular shell or prismatic elements, and 980 elements for quadrilateral shell or hexahedral elements (see Figure 29 for illustration). In order to determine the appropriate number of through-thickness GIP, three simulations with 2, 3, and 5 through-thickness GIP, successively, are first conducted with the SHB-EXP elements. 


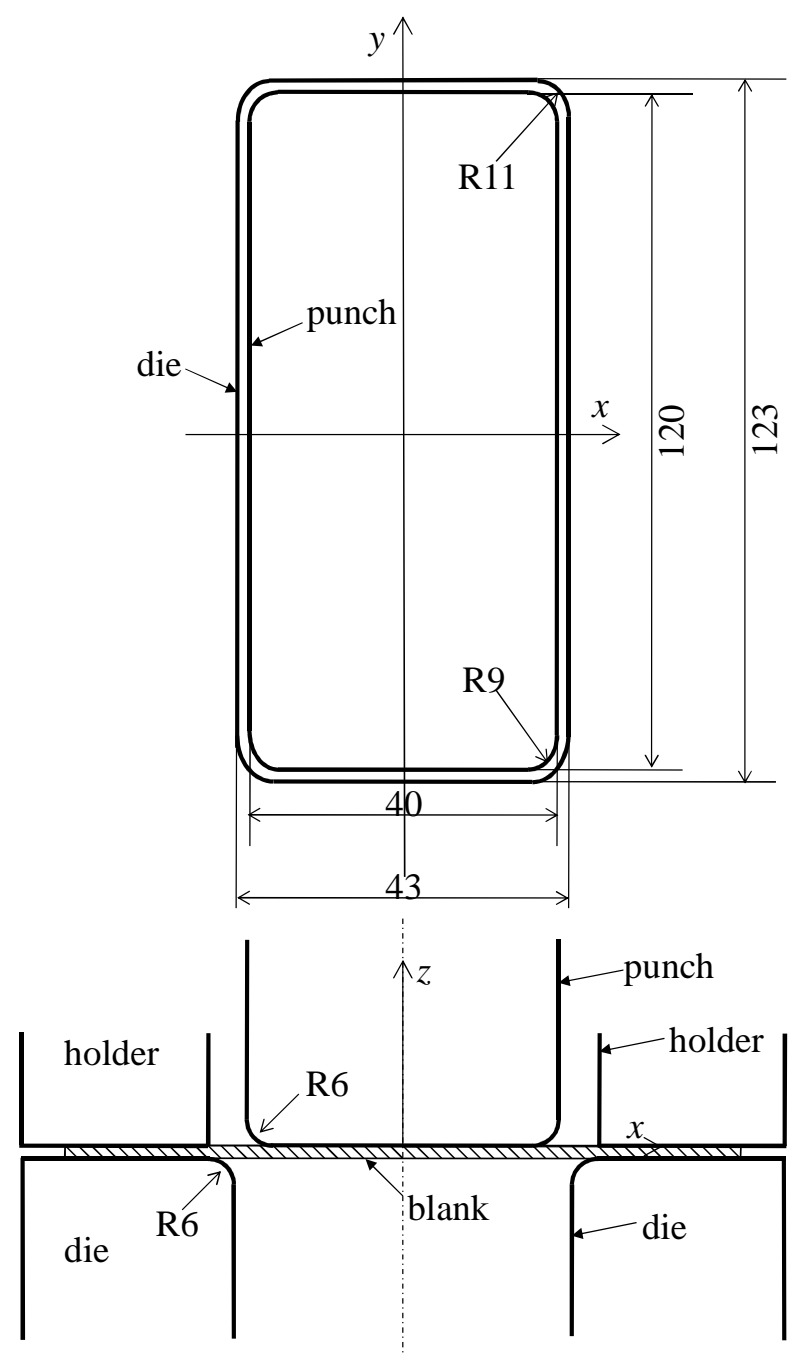

Figure 28. Schematic view for the rectangular cup drawing setup

(a)

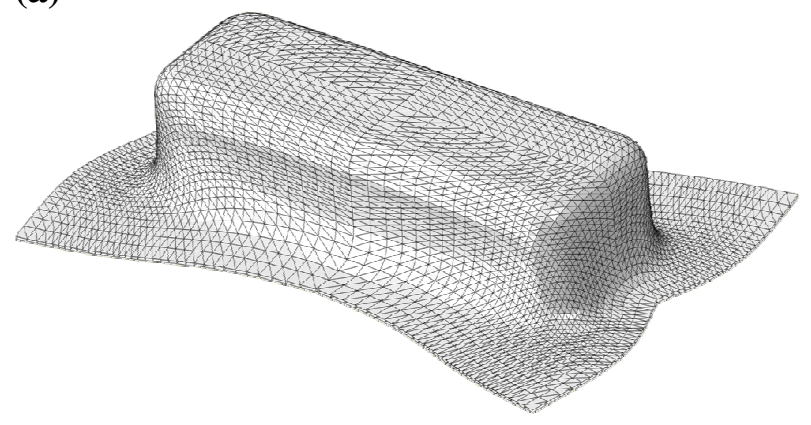

(b)

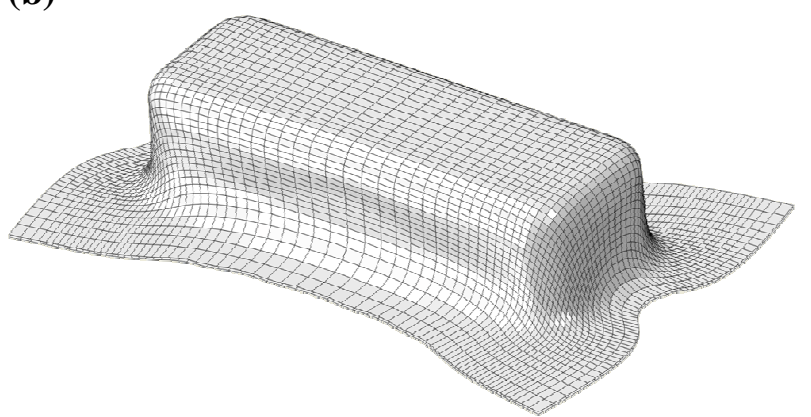

Figure 29. Final deformed mesh for the rectangular cup at a punch stroke of $30 \mathrm{~mm}$ : using (a) SHB6-EXP elements, and (b) using SHB8PS-EXP elements 
Figure 30 shows the predictions of flange contour of the sheet at different punch stroke $(10,20$, and $30 \mathrm{~mm}$ ), as obtained with the SHB-EXP elements using different numbers of throughthickness GIP. These numerical predictions are also compared with the experimental flange contours given by Choi and Huh (1999). Overall, the flange contours predicted by both of the SHB6-EXP and SHB8PS-EXP elements are in good agreement with those measured experimentally for the three forming stages (i.e., 10, 20, and $30 \mathrm{~mm}$ punch stroke). More specifically, for a small punch stroke corresponding to a moderate forming stage (i.e., $10 \mathrm{~mm}$ ), the flange contours obtained with different numbers of through-thickness GIP are indistinguishable and, therefore, 2 GIP appear to be sufficient to obtain accurate results. However, for higher punch stroke corresponding to a deeper forming stage (e.g., $30 \mathrm{~mm}$ ), at least 5 through-thickness GIP are needed to better describe the various non-linear phenomena involved in deep forming processes. This issue has already been discussed in Abed-Meraim and Combescure (2009), where the quasistatic version of the SHB8PS element has been validated in the framework of non-linear quasistatic analysis including elastic-plastic applications.

(a)

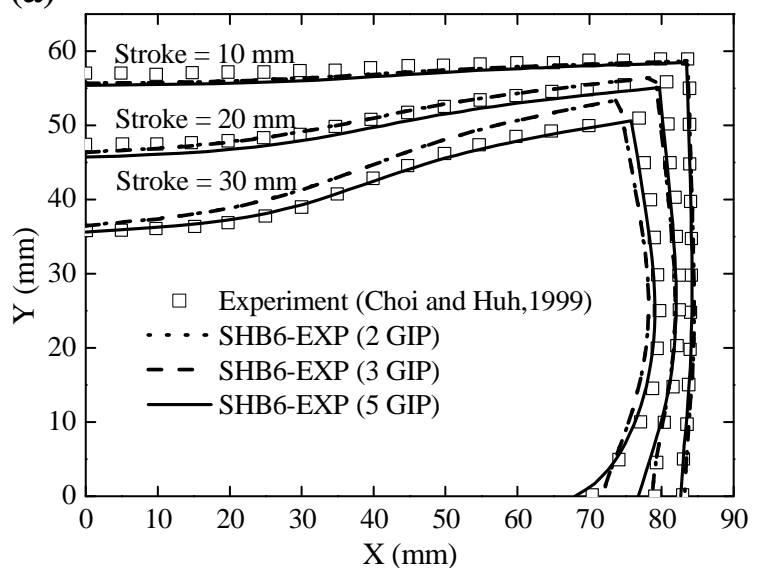

(b)

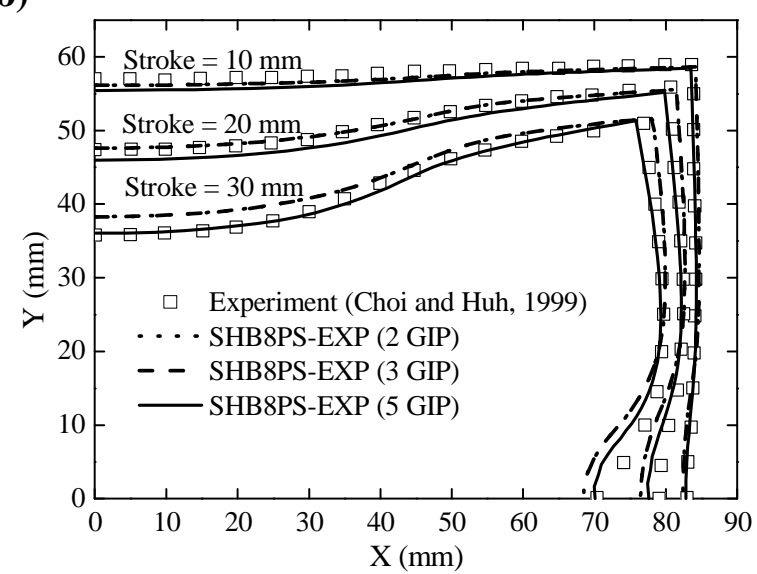

Figure 30. Comparison between the SHB-EXP simulation results and the experiments in terms of flange contour predictions at different punch strokes: influence of the number of through-thickness GIP: using (a) SHB6-EXP elements, and (b) using SHB8PS-EXP elements

Taking the above preliminary analysis into consideration, the simulation results given by the SHB-EXP elements with 5 through-thickness GIP are compared in Figure 31 with those yielded by ABAQUS elements, using the same in-plane mesh and number of through-thickness GIP. Note 
that for the C3D6 and C3D8R ABAQUS solid elements, 5 element layers are required, which leads to 9800 elements and 4900 elements, respectively. Figure 31 shows the flange contour predictions at different punch stroke, as obtained with the SHB-EXP and ABAQUS elements, along with the experimental measurements. It can be seen that, in the case of small punch stroke (i.e., punch stroke of $10 \mathrm{~mm}$ ), the flange contours predicted with the SHB-EXP elements and ABAQUS elements are quite equivalent, whereas the SHB-EXP elements provide the closest results to the experiments in the case of deep forming (i.e., punch stroke of $30 \mathrm{~mm}$ ). These results clearly demonstrate the capability of the proposed SHB-EXP elements of accurately describing the various through-thickness phenomena using only a single element layer with few GIP.

(a)

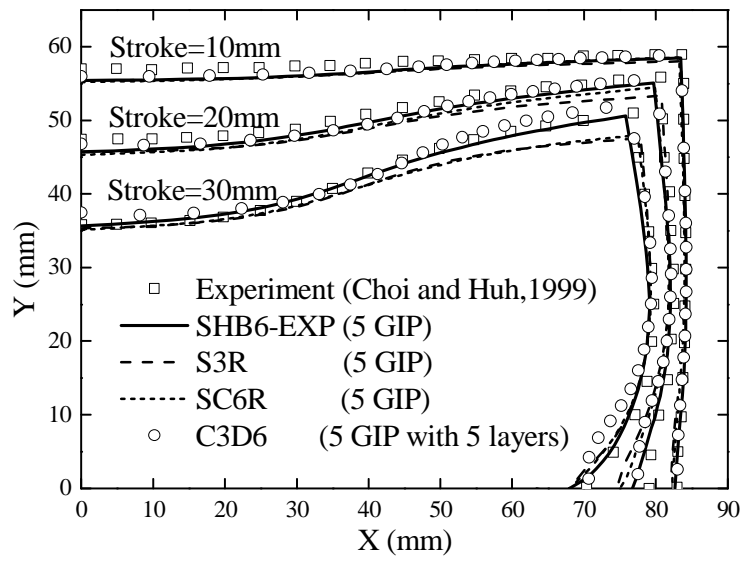

(b)

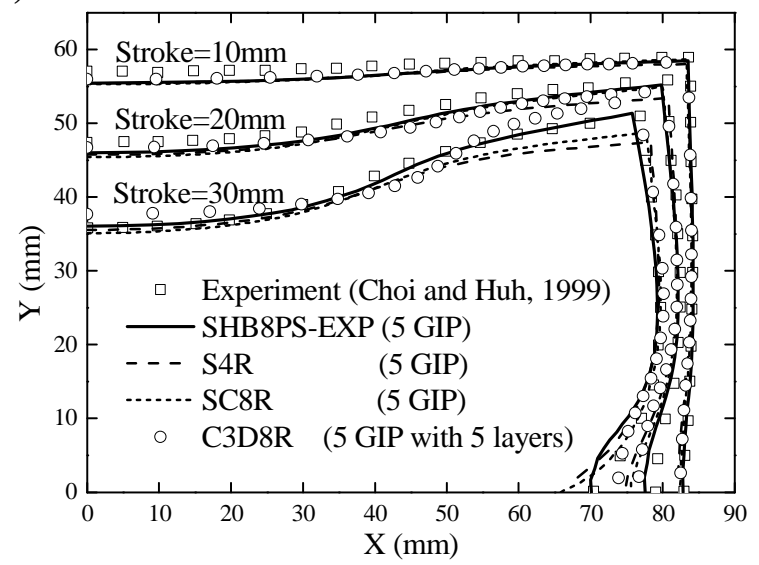

Figure 31. Prediction of flange contours at different punch strokes: comparison between the SHBEXP simulation results using 5 through-thickness GIP, ABAQUS element results, and experimental measurements; (a) triangular shell / prismatic elements, and (b) quadrilateral shell / hexahedral elements

\subsection{Deep drawing of a cylindrical cup}

The deep drawing of cylindrical cup is another popular benchmark test commonly used to study the earing evolution after forming, when the anisotropic plastic behavior of sheet metals is considered. In the present test, deep drawing of a circular plate made of an AA2090-T3 aluminum alloy is investigated. The initial diameter and thickness of the sheet metal are equal to $158.76 \mathrm{~mm}$ and $1.6 \mathrm{~mm}$, respectively. All additional details regarding the process simulation and the experimental results are taken from Yoon et al. (2006). The Swift law (see Table 1) is considered 
here to describe the isotropic hardening behavior, while the Hill' 48 yield criterion is adopted to model the anisotropic plasticity of the sheet metal. The corresponding material parameters are summarized in Table 6. The geometry of the drawing setup and the associated dimensions are all illustrated in Figure 32.

Table 6. Material parameters for the AA2090-T3 aluminum alloy.

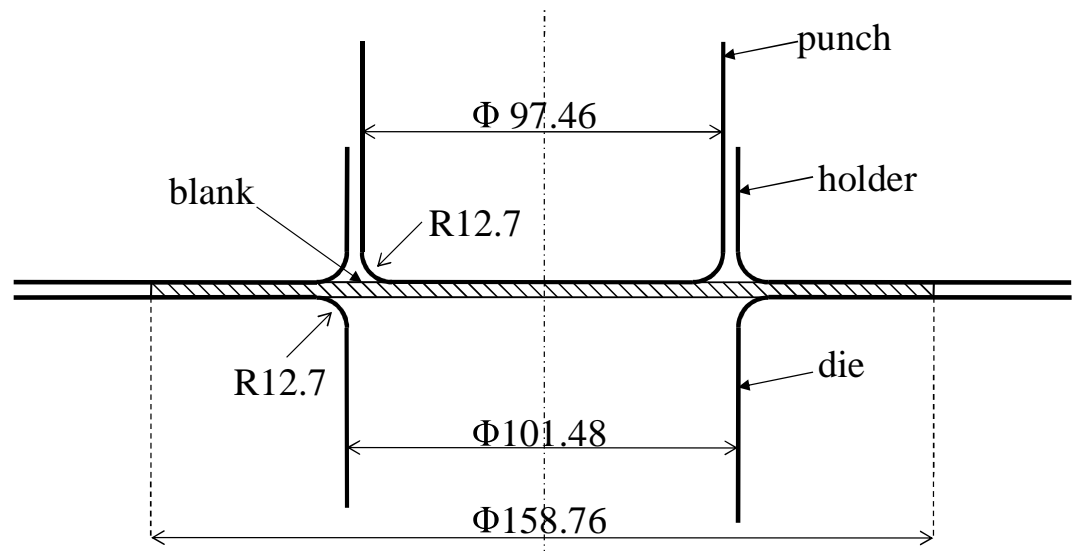

Figure 32. Schematic view for the cylindrical cup drawing setup

Due to symmetry considerations, only one quarter of the circular sheet is modeled, with an inplane discretization of 1350 elements, in the case of triangular shell or prismatic elements, and 800 elements, in the case of quadrilateral shell or hexahedral elements. A constant holder force of 22.2 $\mathrm{kN}$ is applied during the forming process, and the friction coefficient between the sheet and the forming tools is taken to be equal to 0.1 . The deformed cup, corresponding to the end of the forming operation, is illustrated in Figure 33 for both of the SHB6-EXP and SHB8PS-EXP elements. Similar to the previous deep drawing benchmarks, the influence of the number of through-thickness GIP is also investigated in this test by adopting a single SHB-EXP element layer with 2, 3, and 5 through-thickness GIP, successively. The cup height profile predictions obtained with the SHB-EXP elements and ABAQUS elements are reported in Figure 34, for the quarter model, and compared with the experimental measurements taken from Yoon et al. (2006). On the whole, it can be observed that both the shape and the height of the earing profiles predicted 
with the SHB-EXP elements are in good agreement with the experiments. However, these predictions are slightly underestimated at $0^{\circ}$ and $90^{\circ}$ from the rolling direction, while they are closer to the experiments in the range around the experimental peak value at $50^{\circ}$ from the rolling direction. From the sensitivity study to the number of through-thickness GIP, the SHB8PS-EXP element shows good convergence of the results starting from 2 GIP, while the SHB6-EXP element requires at least 3 through-thickness GIP to provide converged results. Furthermore, Figure 34 shows comparisons between the earing profiles predicted with the SHB-EXP elements and ABAQUS elements, using the same in-plane mesh and a comparable number of through-thickness GIP. It can be clearly observed that the SHB-EXP elements provide the closest predictions to the experiments, for almost the entire range of angles from the rolling direction. However, the current predictions may be improved in future work by adopting more appropriate anisotropic yield criteria for aluminum alloys (see, e.g., Barlat et al. 1991; Barlat et al., 2003; Yoon et al., 2006), which are able to predict more than four earing profiles for the complete circular sheet, as observed experimentally for such materials.

(a)

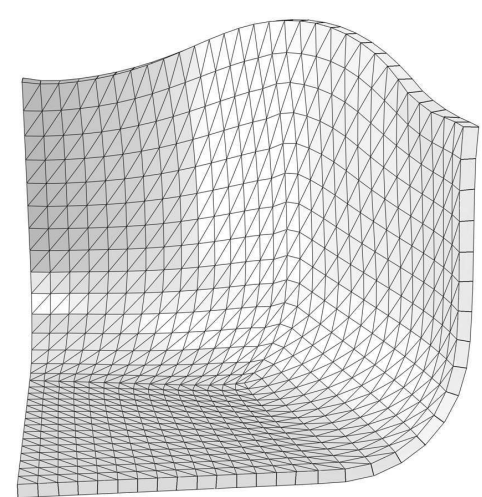

(b)

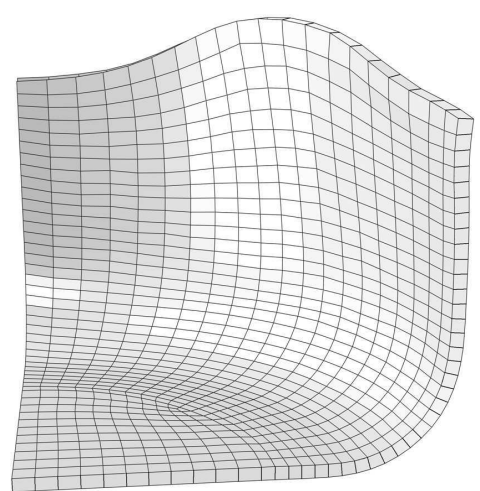

Figure 33. Final deformed mesh for the cylindrical cup: using (a) SHB6-EXP elements, and (b) using SHB8PS-EXP elements 
(a)

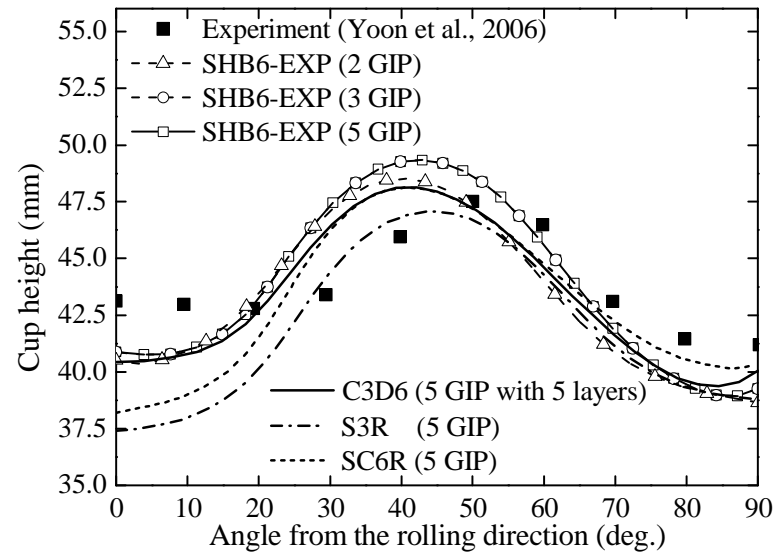

(b)

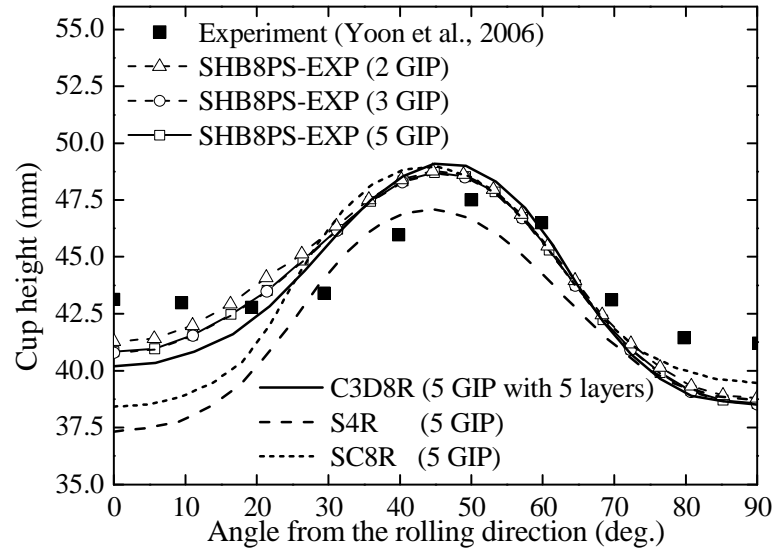

Figure 34. Prediction of cup height profiles: comparison between the SHB-EXP simulation results, ABAQUS element results, and experimental measurements; (a) triangular shell / prismatic elements, and (b) quadrilateral shell / hexahedral elements

\section{Conclusion}

In this paper, two linear solid-shell elements, namely a six-node prismatic element denoted SHB6-EXP and an eight-node hexahedral element denoted SHB8PS-EXP, have been proposed and implemented into the dynamic finite element code ABAQUS/explicit. The main objective of this work is to establish accurate and efficient solid-shell element formulations for the explicit/dynamic analysis of 3D thin structures. These explicit solid-shell versions, denoted SHBEXP, can be applied to structural dynamic analysis, such as impact/crash problems, as well as to complex sheet metal forming processes, for which the use of implicit/quasi-static approaches may exhibit convergence issues.

Several numerical treatments, such as the reduced-integration technique, the assumed-strain method, hourglass mode stabilization, are adopted in the formulation of the SHB-EXP elements to eliminate the main locking phenomena that are inherent in low-order formulations. A characteristic feature is that these elements are based on a fully three-dimensional approach, with only translational degrees of freedom. In addition, they are designed to be used with only a single element layer, while attributing an arbitrary user-defined number of integration points in the thickness direction. This makes them very attractive, due to their computational efficiency in explicit/dynamic analyses. 
The performance of the SHB-EXP elements has been first shown through popular dynamic benchmark tests, involving geometric and material non-linearities. In particular, the SHB-EXP elements demonstrate good capabilities for the modeling of impact-type problems, in both cases of low and high initial velocity of projectiles. The simulation results obtained with the proposed SHB-EXP elements have been validated by comparison with state-of-the-art elements available in ABAQUS, and were also found in good agreement with reference solutions taken from the related literature.

Then, these explicit versions of solid-shell elements have been validated in the context of sheet metal forming applications. Three popular and selective deep drawing processes have been considered in this work, using square, rectangular, and circular anisotropic sheet metals associated with square, rectangular, and cylindrical punch shapes, respectively. These challenging benchmark problems allow evaluating the SHB-EXP elements in severe forming conditions, involving nonlinear loading paths, anisotropic material behavior, and double-sided contact. Using similar meshes and comparable numbers of integration points through the thickness, the comparison between conventional ABAQUS solid elements and the present SHB-EXP elements reveals that the latter provide predictions that are the closest to the experimental results. It is worth noting that the good performance of the SHB-EXP elements is achieved using only a single element layer with few integration points, in contrast to ABAQUS solid elements, which has important consequences on the computational cost. Note also that some convergence issues have been encountered with the SC8R ABAQUS solid-shell element for the modeling of the deep drawing of a square cup, which makes the present SHB8PS-EXP solid-shell element very competitive in such benchmark problems.

On the whole, the performance of the SHB8PS-EXP hexahedral element is better than that of the SHB6-EXP prismatic element. However, these two solid-shell element complement each other, as combination of these may prove necessary for the simulation of complex shaped geometries, which cannot be discretized using only hexahedral elements. Similar situations may also be encountered when using free mesh-generation tools, which results in meshes comprising both hexahedral and prismatic elements. 


\section{References}

Abed-Meraim F. and Combescure A. (2002), "SHB8PS - a new adaptive, assumed-strain continuum mechanics shell element for impact analysis", Computers and Structures, Vol. 80 Nos 9-10, pp. 791-803.

Abed-Meraim F. and Combescure A. (2009), "An improved assumed strain solid-shell element formulation with physical stabilization for geometric non-linear applications and elastic-plastic stability analysis", International Journal for Numerical Methods in Engineering, Vol. 80 No 13, pp. 1640-1686.

Balmer H.A. and Witmer E.A. (1964), Theoretical-experimental correlation of large dynamic and permanent deformations of impulsively-loaded simple structures, Air Force Flight Dynamics Laboratory, Rept. FDP-TDR-64-108, Wright Patterson AFB, OH

Barlat F., Brem J.C., Yoon J.W., Chung K., Dick R.E., Lege D.J., Pourboghrat F., Choi S.H. and Chu E. (2003), "Plane stress yield function for aluminum alloy sheets - part 1: theory", International Journal of Plasticity, Vol. 19 No 9, pp. 1297-1319.

Barlat F., Lege D.J. and Brem J.C. (1991), “A six-component yield function for anisotropic materials", International Journal of Plasticity, Vol. 7 No 7, pp. 693-712.

Belytschko T., Bindeman L.P. (1993), “Assumed strain stabilization of the eight node hexahedral element", Computer Methods in Applied Mechanics and Engineering, Vol. 105 No 2, pp. 225-260.

Belytschko T., Lin J.I. and Tsay C.S. (1984), "Explicit algorithms for the nonlinear dynamics of shells", Computer Methods in Applied Mechanics and Engineering, Vol. 42 No 2, pp. 225 251.

Belytschko T., Wong B.L. and Chiang H.Y. (1992), “Advances in one-point quadrature shell elements", Computer Methods in Applied Mechanics and Engineering, Vol. 96, pp. 93-107.

Cardoso R.P.R., Yoon J.W., Mahardika M., Choudhry S., Alves de Sousa R.J. and Fontes Valente R.A. (2008), "Enhanced assumed strain (EAS) and assumed natural strain (ANS) methods for one-point quadrature solid-shell elements", International Journal for Numerical Methods in Engineering, Vol. 75 No 2, pp. 156-187. 
Chen L.B., Xi F. and Yang J.L. (2007), "Elastic-plastic contact force history and response characteristics of circular plate subjected to impact by a projectile", Acta Mechanica Sinica, Vol. 23 No 4, pp. 415-425.

Cho C., Park H.C. and Lee S.W. (1998), "Stability analysis using a geometrically nonlinear assumed strain solid shell element model”, Finite Elements in Analysis and Design, Vol. 29 No 2, pp. 121-135.

Choi T.H. and Huh H. (1999), "Sheet metal forming analysis of planar anisotropic materials by a modified membrane finite element method with bending effect", Journal of Materials Processing Technology, Vol. 89-90, pp. 58-64.

Edem I.B. and Gosling P.D. (2013), "Physically stabilised displacement-based ANS solid-shell element", Finite Element in Analysis and Design, Vol. 74, pp. 30-40.

Flores F.G. (2013), "Development of a non-linear triangular prism solid-shell element using ANS and EAS techniques", Computer Methods in Applied Mechanics and Engineering, Vol. 266, pp. 81-97.

Guo Y.Q., Gati W., Naceur H. and Batoz J.L. (2002), “An efficient DKT rotation free shell element for springback simulation in sheet metal forming", Computers and Structures, Vol. 80 Nos 27-30, pp. 2299-2312.

Hallquist J.O. (1983), Theoretical manual for DYNA3D, Report UC1D-19041, Lawrence Livermore National Laboratory, Livermore, CA, USA

Hauptmann R. and Schweizerhof K. (1998), “A systematic development of 'Solid-shell' element formulations for linear and non-linear analyses employing only displacement degrees of freedom”, International Journal for Numerical Methods in Engineering, Vol. 42 No 1, pp. 49-69.

Hill R. (1948), "A theory of the yielding and plastic flow of anisotropic metals", Proc. Roy. Soc. London A, Vol. 193 No 1033, pp. 281-297.

Huh H. and Choi T.H. (1999), "Modified membrane finite element formulation for sheet metal forming analysis of planar anisotropic materials", International Journal of Mechanical Sciences, Vol. 42 No 8, pp. 1623-1643. 
Huh H., Han S.S. and Yang D.Y. (1994), "Modified membrane finite element formulation considering bending effects in sheet metal forming analysis", International Journal of Mechanical Sciences, Vol. 36 No 7, pp. 659-671.

Li L.M., Peng Y.H. and Li D.Y. (2011), “A stabilized underintegrated enhanced assumed strain solid-shell element for geometrically nonlinear plate/shell analysis", Finite Element in Analysis and Design, Vol. 47 No 5, pp. 511-518.

Lu H., Ito K., Kazama K. and Namura S. (2006), "Development of a new quadratic shell element considering the normal stress in the thickness direction for simulating sheet metal forming", Journal of Materials Processing Technology, Vol. 171 No 3, pp. 341-347.

Makinouchi A., Nakamachi E., Oñate E. and Wagoner R.H. (1993), "NUMISHEET’93”, Proceedings of the 2nd International Conference and Workshop on Numerical Simulation of $3 D$ Sheet Metal Forming Processes - Verification of Simulation with Experiment, Isehara, Japan

Mars J., Wali M., Jarraya A., Dammak F. and Dhiab A. (2015), "Finite element implementation of an orthotropic plasticity model for sheet metal in low velocity impact simulations", ThinWalled Structures, Vol. 89, pp. 93-100.

Olovsson L., Unosson M. and Simonsson K. (2004), "Selective mass scaling for thin walled structures modeled with tri-linear solid elements", Computational Mechanics, Vol. 34 No 2, pp. 134-136.

Pagani M., Reese S. and Perego U. (2014), “Computationally efficient explicit nonlinear analyses using reduced integration-based solid-shell finite elements", Computer Methods in Applied Mechanics and Engineering, Vol. 268, pp. 141-159.

Parente M.P.L., Fontes Valente R.A., Natal Jorge R.M., Cardoso R.P.R. and Alves de Sousa R.J. (2006), "Sheet metal forming simulation using EAS solid-shell finite elements", Finite Element in Analysis and Design, Vol. 42 No 13, pp. 1137-1149.

Puso M.A. (2000), “A highly efficient enhanced assumed strain physically stabilized hexahedral element", International Journal for Numerical Methods in Engineering, Vol. 49 No 8, pp. 1029-1064. 
Reese S. (2007), “A large deformation solid-shell concept based on reduced integration with hourglass stabilization", International Journal for Numerical Methods in Engineering, Vol. 69 No 8, pp. 1671-1716.

Schwarze M. and Reese S. (2009), "A reduced integration solid-shell finite element based on the EAS and the ANS concept-Geometrically linear problems", International Journal for Numerical Methods in Engineering, Vol. 80 No 10, pp. 1322-1355.

Schwarze M., Vladimirov I.N. and Reese S. (2011), "Sheet metal forming and springback simulation by means of a new reduced integration solid-shell finite element technology", Computer Methods in Applied Mechanics and Engineering, Vol. 200 Nos 5-8, pp. 454-476.

Simo J.C. and Hughes T.J.R. (1986), "On the variational foundations of assumed strain methods", Journal of Applied Mechanics, Vol. 53 No 1, pp. 51-54.

Sukhomlinov L.G., Engelsberg V.K. and Davydov V.N. (1992), “A finite element membrane model for the analysis of axisymmetric sheet metal forming processes", International Journal of Mechanical Sciences, Vol. 34 No 3, pp. 179-193.

Sze K.Y. and Yao L.Q. (2000), “A hybrid stress ANS solid-shell element and its generalization for smart structure modelling. Part I-solid-shell element formulation", International Journal for Numerical Methods in Engineering, Vol. 48 No 4, pp. 545-564.

Taylor R.L., Beresford P.J. and Wilson E.L. (1976), “A non-conforming element for stress analysis", International Journal for Numerical Methods in Engineering, Vol. 10, pp. 12111219.

Trinh V., Abed-Meraim F. and Combescure A. (2011), "A new assumed strain solid-shell formulation "SHB6" for the six-node prismatic finite element", Journal of Mechanical Science and Technology, Vol. 25 No. 9, pp. 2345-2364.

Wilson E.L and Ibrahimbegovic A. (1990), "Use of incompatible displacement modes for the calculation of element stiffnesses or stresses", Finite Elements in Analysis and Design, Vol. 7, pp. 229-241.

Wu T.Y. (2013), "Dynamic nonlinear analysis of shell structures using a vector form intrinsic finite element", Engineering Structures, Vol. 56, pp. 2028-2040. 
Yoon J.W., Barlat F., Dick R.E. and Karabin M.E. (2006), "Prediction of six or eight ears in a drawn cup based on a new anisotropic yield function”, International Journal of Plasticity, Vol. 22 No 1, pp. 174-193.

Zienkiewicz O.C., Taylor R.L. and Zhu J.Z. (2006), The Finite Element Method, sixth ed., Elsevier Ltd. 